\title{
Knots with small rational genus
}

\author{
Danny Calegari and Cameron Gordon
}

\begin{abstract}
If $K$ is a rationally null-homologous knot in a 3-manifold $M$, the rational genus of $K$ is the infimum of $-\chi(S) / 2 p$ over all embedded orientable surfaces $S$ in the complement of $K$ whose boundary wraps $p$ times around $K$ for some $p$ (hereafter: $S$ is a $p$-Seifert surface for $K$ ). Knots with very small rational genus can be constructed by "generic" Dehn filling, and are therefore extremely plentiful. In this paper we show that knots with rational genus less than $1 / 402$ are all geometric - i.e. they may be isotoped into a special form with respect to the geometric decomposition of $M$ - and give a complete classification. Our arguments are a mixture of hyperbolic geometry, combinatorics, and a careful study of the interaction of small $p$-Seifert surfaces with essential subsurfaces in $M$ of non-negative Euler characteristic.
\end{abstract}

Mathematics Subject Classification (2010). 57M27.

Keywords. Knots, rational genus, stable commutator length, Thurston norm, Berge conjecture.

\section{Introduction}

Let $K$ be an oriented knot in a 3-manifold $M$. If $K$ is null-homologous, it bounds an embedded oriented surface $S$, called a Seifert surface. The least genus of such a surface is called the genus of $K$, and is denoted by $g(K)$. More generally, define the rational genus of $K$, denoted by $\|K\|$, to be the infimum of $-\chi(S) / 2 p$ over all embedded orientable surfaces $S$ in the complement of $K$ without disk or sphere components, whose boundary wraps $p$ times around $K$ for some $p$ (a precise definition is given in $\$ 2$ ).

Largely because of an approach to the Berge conjecture via Knot Floer Homology, there has been recent interest in the question of finding knots in 3-manifolds with the property that they are the unique knot in their homology class with least rational genus. Since Knot Floer Homology detects the Thurston norm, and therefore the rational genus of a knot (see Ozsváth-Szabó [18]) such knots have the property that they are characterized by their Knot Floer Homology, and one can study such knots and surgeries on them using the surgery exact sequence. For example, the unknot in $S^{3}$ is the unique knot of genus 0 , and various families of so-called "simple knots" in lens spaces are the unique knots of minimal rational genus in their respective 
homology classes (see Baker [2], Hedden [15] or Rasmussen [19]). Other authors, e.g. [3], have studied rational linking number, and its relation to contact geometry.

One way to produce knots of small rational genus is by surgery. For example, let $K^{\prime}$ be a non-trivial knot in $S^{3}$, and let $M$ be the 3-manifold obtained by $p / q$ surgery on $K^{\prime}$. Let $K$ in $M$ be the core of the surgery solid torus. Then $[K]$ has order $p$ in $H_{1}(M)$, and $\|K\| \leq g\left(K^{\prime}\right) / p-1 / 2 p$ where $g\left(K^{\prime}\right)$ denotes the (ordinary) genus of $K^{\prime}$, since the boundary of a Seifert surface for $K^{\prime}$ wraps $p$ times around $K$ in the surgered manifold. For more detailed examples, see $\$ 2.4$.

The purpose of this paper is firstly to initiate a systematic study of rational genus and some of its properties, and secondly to demonstrate that there is a universal positive constant such that knots in 3-manifolds with rational genus bounded above by this constant can be completely classified. The precise statement of this classification, and the best estimate for the relevant constant, falls into several cases depending on the geometric decomposition of $M$.

In the generic (i.e. hyperbolic) case, the strategy is to deduce information about $K$ in two steps:

$$
L^{1} \text {-homology } \longrightarrow \text { homotopy } \longrightarrow \text { isotopy } .
$$

An estimate for the rational genus of $K$ is really an estimate of the $L^{1}$-norm of a certain relative homology class; such an estimate can be reinterpreted dually in terms of bounded cohomology. Low-dimensional bounded cohomology in hyperbolic manifolds is related to geometry (at the level of $\pi_{1}$ ) by the methods of Calegari [7]. Homotopy information is promoted to isotopy information by drilling and filling, using uniform geometric estimates due to Hodgson-Kerckhoff [16], and Gauss-Bonnet. One interesting technical aspect of the argument is that it involves finding a CAT $(-1)$ representative of a surface in the complement of the cone locus of a hyperbolic cone manifold. Such a surface can be found either by the PL wrapping technique of Soma [25], or the shrinkwrapping technique of Calegari-Gabai [9].

The organization of the paper is as follows. \$2 introduces definitions, proves some basic lemmas, and ends with several subsections enumerating examples. §3 introduces and develops the tools that power our combinatorial arguments (which apply especially to knots with non-hyperbolic complements). §4 treats knots with hyperbolic complements, and the arguments are more geometric and analytic. Finally, $\S 5$ assembles all this material, and contains the main theorems and their proofs.

1.1. Statement of results. The classification of knots with (sufficiently) small rational genus falls into several cases, depending on the prime and geometric decomposition of $M$. These theorems are proved in $\S 5$, and reproduced here for the convenience of the reader.

Reducible Theorem (5.1). Let $K$ be a knot in a reducible manifold $M$. Then either (1) $\|K\| \geq 1 / 12$; or 
(2) there is a decomposition $M=M^{\prime} \# M^{\prime \prime}, K \subset M^{\prime}$ and either

(a) $M^{\prime}$ is irreducible, or

(b) $\left(M^{\prime}, K\right)=\left(\mathbb{R} \mathbb{P}^{3}, \mathbb{R} \mathbb{P}^{1}\right) \#\left(\mathbb{R} \mathbb{P}^{3}, \mathbb{R P}^{1}\right)$.

Lens Theorem (5.2). Let $K$ be a knot in a lens space $M$. Then either

(1) $\|K\| \geq 1 / 24$; or

(2) K lies on a Heegaard torus in $M$; or

(3) $M$ is of the form $L(4 k, 2 k-1)$ and $K$ lies on a Klein bottle in $M$ as a nonseparating orientation-preserving curve.

Hyperbolic Theorem (5.9). Let $K$ be a knot in a closed hyperbolic 3-manifold $M$. Then either

(1) $\|K\| \geq 1 / 402$; or

(2) $K$ is trivial; or

(3) $K$ is isotopic to a cable of the core of a Margulis tube.

Small SFS Theorem (5.10). Let $M$ be an atoroidal Seifert fiber space over $S^{2}$ with three exceptional fibers and let $K$ be a knot in $M$. Then either

(1) $\|K\| \geq 1 / 402$; or

(2) $K$ is trivial; or

(3) $K$ is a cable of an exceptional Seifert fiber of $M$; or

(4) $M$ is a prism manifold and $K$ is a fiber in the Seifert fiber structure of $M$ over $\mathbb{R P}^{2}$ with at most one exceptional fiber.

Toroidal Theorem (5.19). Let $M$ be a closed, irreducible, toroidal 3-manifold, and let $K$ be a knot in $M$. Then either

(1) $\|K\| \geq 1 / 402$; or

(2) $K$ is trivial; or

(3) $K$ is contained in a hyperbolic piece $N$ of the JSJ decomposition of $M$ and is isotopic either to a cable of a core of a Margulis tube or into a component of $\partial N$; or

(4) $K$ is contained in a Seifert fiber piece $N$ of the JSJ decomposition of $M$ and either

(A) $K$ is isotopic to an ordinary fiber or a cable of an exceptional fiber or into $\partial N$, or

(B) $N$ contains a copy $Q$ of the twisted $S^{1}$ bundle over the Möbius band and $K$ is contained in $Q$ as a fiber in this bundle structure; 
or

(5) $M$ is a $T^{2}$-bundle over $S^{1}$ with Anosov monodromy and $K$ is contained in a fiber.

The subsections $\S 2.4$ and $\S 2.5$ discuss constructions giving rise to eight families of examples of knots with arbitrarily small rational genus, illustrating that all the possibilities listed in the classification theorems really do occur.

1.2. Acknowledgements. The first author would like to thank Matthew Hedden and Jake Rasmussen for interesting and stimulating talks they gave at Caltech in 2007, which were the inspiration for this paper. He would also like to thank Marty Scharlemann and Yoav Rieck for useful conversations about thin position. The second author would like to thank Constance Leidy and Peter Oszváth for useful comments. Danny Calegari was partially supported by NSF grant DMS 0707130.

\section{2. $p$-Seifert surfaces}

This section standardizes definitions, proves some basic facts about rational genus, and describes how to construct examples of knots with small rational genus, illustrating the significance of the cases we enumerate in our classification theorems.

2.1. Definitions. We formalize definitions in this section. Throughout, all 3-manifolds considered will be compact, connected and orientable. A knot $K$ in a 3-manifold $M$ is a tamely embedded $S^{1}$. If $K$ is null-homologous in $M$, a Seifert surface for $K$ is a connected embedded two-sided surface $S$ in $M$ with $\partial S=K$. The genus of $K$ is the least genus of a Seifert surface.

This can be generalized as follows. By analogy with the Thurston norm on $\mathrm{H}_{2}(M)$, we adopt the following notation:

Notation 2.1. If $S$ is a compact, orientable connected surface, define $\chi^{-}(S):=$ $\min (0, \chi(S))$. If $S$ is a compact, orientable surface with components $S_{i}$, define $\chi^{-}(S)=\sum_{i} \chi^{-}\left(S_{i}\right)$. Denote $\eta(S)=-\chi^{-}(S) / 2$.

Remark 2.2. The normalizing factor of 2 in the denominator of $\eta$ reflects the fact that Euler characteristic is "almost" -2 times genus for a surface with a bounded number of boundary components.

Definition 2.3. Let $K$ be a knot in a 3-manifold $M$, with regular neighborhood $N(K)$. If $p$ is a positive integer, a $p$-Seifert surface for $K$ is a compact, oriented surface $S$ embedded in $M-$ int $N(K)$ such that $S \cap \partial N(K)=\partial S$, and $[\partial S]=p[K] \in$ 
$H_{1}(N(K)$ ) (for some choice of orientation on $K$ ). In this case we define the norm, or rational genus of $K$ by

$$
\|K\|=\inf _{S} \eta(S) / p
$$

where the infimum is taken over all $p$ and all $p$-Seifert surfaces $S$ for $K$.

A $p$-Seifert surface $S$ for $K$ can be extended into $N(K)$ to give a map $S \rightarrow$ $M$ which is an embedding on int $S$ and whose restriction $\partial S \rightarrow K$ is a (possibly disconnected) covering map of degree $p$. We will regard a $p$-Seifert surface for $K$ as a singular surface in $M$ in this way in $\$ 4$.

Remark 2.4. A knot $K$ has a $p$-Seifert surface for some $p$ if and only if $[K]$ has finite order in $H_{1}(M)$.

Definition 2.5. A $p$-Seifert surface is good if it satisfies the following properties:

(1) $S$ is connected.

(2) $S$ is incompressible in $M-$ int $N(K)$.

(3) $\partial S$ consists of $q$ parallel, coherently oriented copies of an essential simple closed curve $\rho$ on $\partial N(K)$ that represents $r$ times a generator of $H_{1}(N(K))$, where $q r=p$.

Lemma 2.6. Let $S$ be a $p$-Seifert surface for $K$. Then there is a good $p^{\prime}$-Seifert surface $S^{\prime}$ for $K$ satisfying

$$
\eta(S) / p \geq \eta\left(S^{\prime}\right) / p^{\prime}
$$

Proof. Boundary components of $S$ that are inessential in $\partial N(K)$ may be capped off with disks, and closed components of $S$ may be discarded; neither of these operations increases $\eta$ or changes $p$. If some component of $S$ is a disk $D$, then $D$ is a good $p^{\prime}$-Seifert surface for $K$ for some $p^{\prime}$, and $\eta(D)$ and $\|K\|$ are both zero. Hence we may assume that every component $S_{i}$ of $S$ satisfies $\chi\left(S_{i}\right)=\chi^{-}\left(S_{i}\right)$, and has non-empty boundary, each component of which is an essential curve in $\partial N(K)$.

Since a $p$-Seifert surface is embedded, the components of $\partial S$ are all parallel in $\partial N(K)$, and are therefore all isotopic (though a priori they might have opposite orientations). Let $S$ be a $p$-Seifert surface for $K$, and suppose there are a pair of adjacent components of $\partial S$ on $\partial N(K)$ that are oppositely oriented. Tubing this pair of components does not affect $\eta(S)$ or $p$, so without loss of generality we may assume that all components are coherently oriented.

If $S$ is compressible in $M-$ int $N(K)$ then compressing it along a disk gives a surface $S^{\prime}$ with $\partial S^{\prime}=\partial S$ and $\eta\left(S^{\prime}\right) \leq \eta(S)$. So we may assume that $S$ is incompressible. 
It remains to show that we can take $S$ to be connected. Suppose $S$ is the disjoint union of $S_{1}$ and $S_{2}$, where $\partial S_{i}$ consists of $q_{i}$ copies of $\rho$, and each $q_{i}>0$. Then we estimate

$$
\frac{\eta(S)}{p}=\frac{\eta(S)}{q r}=\frac{\eta\left(S_{1}\right)+\eta\left(S_{2}\right)}{\left(q_{1}+q_{2}\right) r} \geq \min \left\{\frac{\eta\left(S_{1}\right)}{q_{1} r}, \frac{\eta\left(S_{2}\right)}{q_{2} r}\right\} .
$$

It will be important in the sequel to consider surfaces in manifolds $M$ that meet $\partial M$.

Definition 2.7. A relative $p$-Seifert surface $F$ for a knot $K$ in $M$ is an oriented surface, properly embedded in $M-$ int $N(K)$ such that $[\partial F \cap \partial N(K)]=p[K] \in H_{1}(N(K))$.

The definition of good extends to relative $p$-Seifert surfaces, and Lemma 2.6 generalizes to such surfaces as well and with the same proof, so in the sequel we assume all our $p$-Seifert surfaces, relative or otherwise, are good.

Notation 2.8. In the sequel, we write $X:=M-$ int $N(K)$.

2.2. Thurston norm. A basic reference for this section is Thurston's paper [26].

If $K$ is a knot in a closed 3-manifold $M$, then $X$ is a compact 3-manifold with torus boundary, and as is well-known, the kernel of the inclusion map

$$
H_{1}(\partial X ; \mathbb{Q}) \rightarrow H_{1}(X ; \mathbb{Q})
$$

is 1-dimensional, and denoted $L$. Consequently, the kernel of

$$
H_{1}(\partial X ; \mathbb{Z}) \rightarrow H_{1}(X ; \mathbb{Z})
$$

is isomorphic to $\mathbb{Z}$, and is generated by a single element $m[\rho]$, where $[\rho]$ is primitive in $H_{1}(\partial X ; \mathbb{Z})$, and $[\rho]$ is represented by a simple loop $\rho \subset \partial X$ which represents $r$ times a generator of $H_{1}(N(K))$ (this is the same $\rho$ as before).

Let $\partial^{-1} L$ denote the subspace of $H_{2}(X, \partial X ; \mathbb{Q})$ that is the preimage of $L$ under the connecting homomorphism in rational homology. If $S$ is a $p$-Seifert surface for $K$, then $p=q r$ where $m \mid q$, and $[S] \in \partial^{-1} L$. Consider the affine rational subspace $\partial^{-1}[\rho] / r \subset \partial^{-1} L$. The multiple $[S] / p \in \partial^{-1}[\rho] / r$, and $\eta(S) / p=\|S\|_{T} / 2 p$ where $\|\cdot\|_{T}$ denotes the Thurston norm of a surface. Hence, by the definition of rational genus and of Thurston norm, we obtain the following formula:

Lemma 2.9. There is an equality

$$
\|K\|=\inf _{A \in \partial^{-1}[\rho] / r}\|A\|_{T} / 2
$$

where $\|A\|_{T}$ denotes the Thurston norm of the (rational) homology class $A$. 
Since $\|\cdot\|_{T}$ is a convex, non-negative, piecewise rational linear function on $H_{2}(X, \partial X ; \mathbb{R})$, the infimum of $\|\cdot\|_{T} / 2$ is achieved (on some rational subpolyhedron) on the rational affine subspace $\partial^{-1}[\rho] / r$. Consequently, we have:

Proposition 2.10. Let $K$ be a knot. The rational genus $\|K\|$ is equal to $\eta(S) / p$ for some $p$-Seifert surface $S$ and some $p$. Therefore $\|K\|$ is rational. Moreover, there is an algorithm to find $S$ and compute $\|K\|$.

Proof. There is a (straightforward) algorithm to compute the Thurston norm, described in [26], and to find a norm-minimizing surface in any integral class (note that such a surface can be taken to be normal relative to a fixed triangulation, and therefore may be found by linear programming in normal surface space).

Although Proposition 2.10 is included for completeness, it is not essential for the remainder of the paper, and it is generally good enough in the sequel to work with a $p$-Seifert surface that comes close to realizing $\|K\|$.

The first thing one wants to know about an invariant is when it vanishes.

Theorem 2.11. Let $K$ be a knot in a 3-manifold $M$. Then $\|K\|=0$ if and only if either

(1) $K$ bounds a disk in $M$; or

(2) $K$ is the core of a genus 1 Heegaard splitting of a lens space summand of $M$; or

(3) $K$ is the fiber of multiplicity $r$ in a Seifert fiber subspace of $M$ whose base orbifold is a Möbius band with one orbifold point of order $r \geq 1$.

Proof. Suppose $\|K\|=0$. Then by Lemma 2.6 and Proposition 2.10, $K$ has a good $p$-Seifert surface with $\chi^{-}(S)=0$, i.e. $S$ is a disk or annulus.

First assume $S$ is a disk. If $p=1$ we have conclusion (1). If $p>1$ then a regular neighborhood of $N(K) \cup S$ is a punctured lens space with fundamental group $\mathbb{Z} / p \mathbb{Z}$, with $K$ as a core of a genus 1 Heegaard splitting.

If $S$ is an annulus, note that both boundary components of $S$ wrap with the same orientation $r$ times around $K$. A regular neighborhood of $N(K) \cup S$ evidently has the desired structure.

Remark 2.12. Since $M$ is orientable, the total space of the Seifert fiber subspace in bullet (3) of Theorem 2.11 is orientable; i.e. it is a twisted $S^{1}$ bundle over an orbifold Möbius band. There is no suggestion that it is essential in $M$. The situation described in (3) arises in case (2) (b) of Theorem 5.1, case (3) of Theorem 5.2, case (4) of Theorem 5.10, and case (4) (B) of Theorem 5.19.

Under suitable homological conditions on $M$ and $K$, the analysis simplifies considerably: 
Lemma 2.13. Let $K$ be a knot in a $\mathbb{Q}$-homology 3-sphere and let $S$ be a connected $p$-Seifert surface for $K$. Then $p$ is the order of $[K]$ in $H_{1}(M)$.

Proof. Since $M$ is a $\mathbb{Q}$-homology sphere, $H_{2}(X)=0$ and hence the boundary map $H_{2}(X, \partial X) \rightarrow H_{1}(\partial X)$ is injective, with image $\operatorname{ker}\left(H_{1}(\partial X) \rightarrow H_{1}(X)\right) \cong \mathbb{Z}$. Let $x$ be a generator of $H_{2}(X, \partial X)$. Then the image of $x$ under the composition

$$
H_{2}(X, \partial X) \rightarrow H_{1}(\partial X) \rightarrow H_{1}(N(K)) \cong \mathbb{Z}
$$

is $p_{0}$, say, the order of $[K]$ in $H_{1}(M)$. Let $S$ be a connected $p$-Seifert surface for $K$. Then $[S]=k x$ and $p=k p_{0}$ for some $k>0$. But since $S$ is connected, $k=1$ by Lemma 1 of [26].

If $K$ is a knot in a homology 3-sphere, the (ordinary) genus of $K$, denoted by $g(K)$, is the minimal genus of any Seifert surface for $K$. Lemma 2.13 reduces the study of rational genus to that of the usual genus in homology spheres:

Corollary 2.14. If $K$ is a knot in a homology 3-sphere then

$$
\|K\|= \begin{cases}0, & \text { if } g(K)=0 \\ g(K)-1 / 2, & \text { if } g(K)>0\end{cases}
$$

Proof. By Lemma 2.13, $\|K\|=\eta(S)$, where $S$ is a minimal genus Seifert surface for $K$. If $S$ is a disk, $\|K\|=g(K)=0$. Otherwise, $\eta(S)=(2 g(S)-1) / 2=$ $g(S)-1 / 2$.

The following lemma will allow us to construct knots in 3-manifolds with arbitrarily small (non-zero) rational genus.

Lemma 2.15. Let $K^{\prime}$ be a knot in a homology 3-sphere $M^{\prime}$. Let $M$ be the manifold obtained by $m / n$-Dehn surgery on $K^{\prime}$, where $m>0$, and let $K \subset M$ be the core of the surgery solid torus. Then $\|K\|=\left\|K^{\prime}\right\| / m$.

Proof. Note that $[K]$ has order $m$ in $H_{1}(M)=\mathbb{Z} / m \mathbb{Z}$.

Let $S$ be a good $p$-Seifert surface for $K$ in $M$ such that $\|K\|=\eta(S) / p$. The restriction of $S$ to $M-K=M^{\prime}-K^{\prime}$ extends to a good $p^{\prime}$-Seifert surface for $K^{\prime}$ (which by abuse of notation we call $S$ ) where $p^{\prime}=p / m$. By the proof of Lemma $2.13, p^{\prime}=1$ and $S$ is a Seifert surface for $K^{\prime}$ in $M^{\prime}$. Conversely a Seifert surface for $K^{\prime}$ can also be thought of as a good $m$-Seifert surface for $K$. Therefore $\left\|K^{\prime}\right\|=\eta(S)$ and $p=m$. Hence $\|K\|=\left\|K^{\prime}\right\| / m$. 
2.3. Connect sums. We now examine the behavior of rational genus under connected sum. In this context it is convenient to say that a knot $K$ in $M$ is $p$-trivial if it has a $p$-Seifert surface that is a disk. If $p=1$ then $K$ bounds a disk, and is trivial. If $p>1$ then $K$ is the core of a genus 1 Heegaard splitting of a lens space summand of $M$ with fundamental group $\mathbb{Z} / p \mathbb{Z}$.

Remark 2.16. If $K$ is $p$-trivial for some $p$ then clearly $\partial N(K)$ is compressible in $X$. Conversely, a compressing disk for $\partial N(K)$ in $X$ is either a $p$-Seifert surface for $K$, for some $p \geq 1$, or has boundary a meridian of $K$, in which case $K$ is isotopic to $S^{1} \times$ \{point in some $S^{1} \times S^{2}$ summand of $M$. So for rationally null-homologous knots, being $p$-trivial for some $p$ is equivalent to $\partial N(K)$ being compressible in $X$.

Theorem 2.17. Let $K_{1}$ and $K_{2}$ be knots in 3-manifolds $M_{1}$ and $M_{2}$. Then

(1) if $K_{1}$ is $p_{1}$-trivial and $K_{2}$ is trivial then $K_{1} \# K_{2}$ is $p_{1}$-trivial;

$$
\left\|K_{1} \# K_{2}\right\|=\left\{\begin{array}{l}
\left\|K_{1}\right\|+\left\|K_{2}\right\|+\frac{1}{2} \text { if } K_{1} \text { and } K_{2} \text { are not } p \text {-trivial for any } p \\
\left\|K_{1}\right\|+\frac{1}{2}-\frac{1}{2 p_{2}} \text { if } K_{2} \text { is } p_{2} \text {-trivial, } K_{1} \text { is not } p \text {-trivial for any } p \\
\frac{1}{2}-\frac{\left(p_{1}+p_{2}\right)}{2 p_{1} p_{2}} \text { if } K_{i} \text { is } p_{i} \text {-trivial, } p_{i} \geq 2, i=1,2 .
\end{array}\right.
$$

Remark 2.18. The first case in bullet (2) says that for knots that are not $p$-trivial for any $p$, the quantity $\|K\|+\frac{1}{2}$ is additive under connected sum, and is the analog of the additivity of genus for knots in $S^{3}$; see Corollary 2.14 .

Remark 2.19. Theorem 2.17 has an analog in the theory of stable commutator length (see Definition 4.1); compare with the Product formula (Theorem 2.93) from [8].

Remark 2.20. Note that $K$ is 2-trivial if and only if $K$ is contained in an $\mathbb{R} \mathbb{P}^{3}$ summand as $\mathbb{R P}^{1}$. Also, it follows from Theorem 2.17 that if $K=K_{1} \# K_{2}$ with $K_{1}$ and $K_{2}$ non-trivial, then $\|K\|=0$ if and only if $K_{1}$ and $K_{2}$ are 2-trivial, i.e. $K$ is contained in an $\mathbb{R} \mathbb{P}^{3} \# \mathbb{R} \mathbb{P}^{3}$ summand as $\mathbb{R} \mathbb{P}^{1} \# \mathbb{R} \mathbb{P}^{1}$. This is a special case of Theorem 2.11 (3), with $r=1$.

Proof of Theorem 2.17. Let $S_{i} \subset M_{i}$ be a good $p_{i}$-Seifert surface for $K_{i}$ with $\left\|K_{i}\right\|=\eta\left(S_{i}\right) / p_{i}, i=1,2$. Then we may construct a $p_{1} p_{2}$-Seifert surface $S$ for $K=K_{1} \# K_{2}$ in $M_{1} \# M_{2}$ by taking $p_{2}$ copies of $S_{1}$ and $p_{1}$ copies of $S_{2}$ and joining them along $p_{1} p_{2}$ arcs. We have

$$
\chi(S)=p_{2} \chi\left(S_{1}\right)+p_{1} \chi\left(S_{2}\right)-p_{1} p_{2} .
$$


Conversely, let $S$ be a good $p$-Seifert surface for $K=K_{1} \# K_{2}$ with $\|K\|=\eta(S) / p$. Suppose $\partial S$ has $q$ components, each having intersection number $r$ with a meridian of $K$. Let $A$ be the annulus in $X=M_{1} \# M_{2}-$ int $N(K)$ that realizes the connected sum decomposition. By an isotopy of $S$ we may assume that each component of $\partial S$ meets each component of $\partial A$ in $r$ points, and that $S \cap A$ is a disjoint union of arcs and simple closed curves. Since the boundary components of $S$ are oriented coherently on $\partial N(K)$, each arc must have one endpoint on each component of $\partial A$. It follows that any simple closed curve of intersection is inessential in $A$, and therefore in $S$, and so these can be removed by performing surgery on $S$ and discarding the resulting 2-spheres. Hence we may assume that $S \cap A$ consists of $q r=p$ essential $\operatorname{arcs}$ in $A$. Cutting $S$ along these arcs gives $p$-Seifert surfaces $S_{1}, S_{2}$ for $K_{1}, K_{2}$ in $M_{1}, M_{2}$. Note that

$$
\chi(S)=\chi\left(S_{1}\right)+\chi\left(S_{2}\right)-p .
$$

To prove part (1), note that if $S_{1}$ and $S_{2}$ are disks and $p_{2}=1$ then the $p_{1}$-Seifert surface for $K_{1} \# K_{2}$ in (2.3.1) is a disk.

To prove (2), first suppose that $K_{1}$ and $K_{2}$ are not $p$-trivial (for any $p$ ). Then the surfaces $S_{1}$ and $S_{2}$ in (2.3.1) and (2.3.2) have no disk components, and from (2.3.1) we get

$$
\eta(S)=p_{2} \eta\left(S_{1}\right)+p_{1} \eta\left(S_{2}\right)+p_{1} p_{2} / 2
$$

and hence

$$
\begin{aligned}
\|K\| \leq \eta(S) / p_{1} p_{2} & =\eta\left(S_{1}\right) / p_{1}+\eta\left(S_{2}\right) / p_{2}+\frac{1}{2} \\
& =\left\|K_{1}\right\|+\left\|K_{2}\right\|+\frac{1}{2} .
\end{aligned}
$$

Similarly, (2.3.2) gives

$$
\eta(S)=\eta\left(S_{1}\right)+\eta\left(S_{2}\right)+p / 2
$$

and hence

$$
\begin{aligned}
\|K\|=\eta(S) / p & =\eta\left(S_{1}\right) / p+\eta\left(S_{2}\right) / p+\frac{1}{2} \\
& \geq\left\|K_{1}\right\|+\left\|K_{2}\right\|+\frac{1}{2} .
\end{aligned}
$$

Together, (2.3.3) and (2.3.4) give the first assertion in part (2) of the theorem.

Second, suppose $K_{2}$ is $p_{2}$-trivial and $K_{1}$ is not $p$-trivial for any $p$. Then in (2.3.1) $S_{2}$ is a disk while $\chi\left(S_{1}\right) \leq 0$. Hence $\chi(S) \leq 0$, and we get

$$
\begin{aligned}
\|K\| \leq \eta(S) / p_{1} p_{2} & =\eta\left(S_{1}\right) / p_{1}-\frac{1}{2 p_{2}}+\frac{1}{2} \\
& =\left\|K_{1}\right\|-\frac{1}{2 p_{2}}+\frac{1}{2} .
\end{aligned}
$$


In (2.3.2), since $K_{1}$ is not $p$-trivial for any $p$, no component of $S_{1}$ is a disk. Hence $\eta\left(S_{1}\right)=-\chi\left(S_{1}\right) / 2, \chi\left(S_{1}\right) \leq 0$, and $\chi(S) \leq 0$. Let $S_{2}$ have $d_{2}$ disk components. Then $\chi\left(S_{2}\right) \leq d_{2}$ and

$$
d_{2} p_{2} \leq p
$$

Now

$$
\begin{aligned}
\|K\|=\eta(S) / p & =-\chi(S) / 2 p \\
& \geq-\chi\left(S_{1}\right) / 2 p-\frac{d_{2}}{2 p}+\frac{1}{2} \\
& \geq\left\|K_{1}\right\|-\frac{d_{2}}{2 p}+\frac{1}{2} .
\end{aligned}
$$

Comparing (2.3.7) with (2.3.5) we get

$$
\frac{d_{2}}{2 p} \geq \frac{1}{2 p_{2}}, \quad \text { i.e. } \quad d_{2} p_{2} \geq p .
$$

By (2.3.6), this gives $d_{2} p_{2}=p$ and (2.3.5) is an equality.

Finally, suppose that $K_{i}$ is $p_{i}$-trivial, $p_{i} \geq 2, i=1,2$. In (2.3.1), $S_{1}$ and $S_{2}$ are disks, and

$$
\chi(S)=p_{1}+p_{2}-p_{1} p_{2} \leq 0
$$

Hence

$$
\begin{aligned}
\|K\| \leq \eta(S) / p_{1} p_{2} & =\frac{p_{1} p_{2}-\left(p_{1}+p_{2}\right)}{2 p_{1} p_{2}} \\
& =\frac{1}{2}-\frac{\left(p_{1}+p_{2}\right)}{2 p_{1} p_{2}} .
\end{aligned}
$$

Now consider (2.3.2), and let $S_{i}$ have $d_{i}$ disk components, $i=1,2$. Note that

$$
d_{i} p_{i} \leq p, \quad i=1,2 \text {; }
$$

in particular, since $p_{i} \geq 2, d_{i} \leq p / 2, i=1,2$. It follows that

$$
\chi(S) \leq d_{1}+d_{2}-p \leq 0 .
$$

Therefore

$$
\begin{aligned}
\|K\|=\eta(S) / p & =-\chi(S) / 2 p \\
& \geq \frac{1}{2}-\frac{\left(d_{1}+d_{2}\right)}{2 p} .
\end{aligned}
$$

Comparing (2.3.10) with (2.3.8) gives

$$
\frac{d_{1}}{p}+\frac{d_{2}}{p} \geq \frac{1}{p_{1}}+\frac{1}{p_{2}} .
$$

On the other hand, by (2.3.9) we have $d_{i} / p \leq 1 / p_{i}, i=1,2$, and hence (2.3.8) is an equality, as desired. 
2.4. Examples of knots with small rational genus. We use Lemma 2.15 to construct examples of knots $K$ in 3-manifolds $M$ with arbitrarily small (but non-zero) rational genus. These examples illustrate cases from our main classification theorems, to be proved in $\$ 4$ and $\S 5$, especially case (2) of Theorem 4.8, case (2) of Theorem 5.2, case (3) of Corollary 5.9, case (3) of Theorem 5.10, and cases (3) and (4) of Theorem 5.19. We recall the notation $\Delta(\cdot, \cdot)$ for the minimal number of points of intersection (i.e. the homological intersection number) of two unoriented isotopy classes of simple essential loops on a torus; in co-ordinates, $\Delta(a / b, c / d)=|a d-b c|$.

Case A. ( $M$ is hyperbolic and $K$ is the core of a Margulis tube.) Let $K^{\prime}$ be a hyperbolic knot in $S^{3}$. Let $M$ be the result of $m / n$-Dehn surgery on $K^{\prime}, m>0$, and let $K$ be the core of the surgery solid torus. By Lemma 2.15, $\|K\|=\left\|K^{\prime}\right\| / m \rightarrow 0$ as $m \rightarrow \infty$. Also, for $m$ sufficiently large $M$ is hyperbolic and $K$ is a geodesic in $M$ whose length $\rightarrow 0$ as $m \rightarrow \infty$.

Case B. ( $M$ is a lens space and $K$ lies on a genus 1 Heegaard surface for $M$.) Let $(M, K)$ be as in Case A above, but with $K^{\prime}$ a $(u, v)$-torus knot in $S^{3}$, where $u, v>1$. If $d=\Delta(m / n, u v / 1)=|m-n u v|=1$ then $M$ is a lens space, and one sees that $K$ lies on a Heegaard torus in $M$. By choosing $m$ large enough we can make $\|K\|=\left\|K^{\prime}\right\| / m$ arbitrarily small.

Case C. ( $M$ is a Seifert fiber space and $K$ is an ordinary or exceptional fiber.) By taking $d>1$ in Case B above, $M$ becomes a Seifert fiber space with base orbifold $S^{2}$ with three cone points of orders $u, v$ and $d$, and $K$ is the exceptional fiber of multiplicity $d$.

More generally, let $M^{\prime}$ be a Seifert fibered homology 3-sphere, with base $S^{2}$ and $k \geq 3$ exceptional fibers. Let $K^{\prime}$ be an ordinary fiber, and let $a / b$ be the slope of the fiber on $\partial X^{\prime}$, where $X^{\prime}=M^{\prime}-$ int $N\left(K^{\prime}\right)$. Let $M$ be $m / n$-Dehn surgery on $K^{\prime}$, and $K$ the core of the surgery solid torus. Let $d=\Delta(a / b, m / n)=|a n-b m|$. If $d=1$, then $M$ is a Seifert fiber space over $S^{2}$ with $k$ exceptional fibers, and $K$ is an ordinary fiber. If $d>1$, then $M$ is a Seifert fiber space over $S^{2}$ with $(k+1)$ exceptional fibers, and $K$ is an exceptional fiber of multiplicity $d$. By Lemma 2.15, $\|K\|=\left\|K^{\prime}\right\| / m$, and in both cases this can be made arbitrarily small by taking $m$ sufficiently large.

Case D. ( $M$ is hyperbolic and $K$ is a non-trivial cable of the core of a Margulis tube.) Let $K_{0}$ be a hyperbolic knot in $S^{3}$. Fix coprime integers $p, q>1$, and let $k$ be any positive integer. Then $k q^{2}$ and $(1+k p q)$ are coprime, so there exist integers $a, b$ such that $a k q^{2}-b(1+k p q)=1$. Let $M$ be the manifold obtained by $\left(-k q^{2} / b\right)$-Dehn surgery on $K_{0}$, and let $K^{\prime} \subset M$ be the core of the surgery. Let $X_{0}$ be the exterior of $K_{0}$; then $M=X_{0} \cup V$ where $V$ is a tubular neighborhood of $K^{\prime}$. Let $\mu_{0}, \lambda_{0}$ (resp. $\mu, \lambda$ ) be a canonical meridian-longitude pair of generators for $H_{1}\left(\partial X_{0}\right)$ (resp. $H_{1}(\partial V)$ ). Let $f: \partial V \rightarrow \partial X_{0}$ be the gluing homeomorphism. We can choose $f$ so 
that with respect to the above bases $f_{*}: H_{1}(\partial V) \rightarrow H_{1}\left(\partial X_{0}\right)$ is given by the matrix

$$
A=\left(\begin{array}{cc}
k q^{2} & -(1+k p q) \\
-b & a
\end{array}\right)
$$

Then

$$
A^{-1}=\left(\begin{array}{cc}
a & 1+k p q \\
b & k q^{2}
\end{array}\right)
$$

in particular, under the gluing the longitude $\lambda_{0}$ on $\partial X_{0}$ is identified with a curve of slope $(1+k p q) / k q^{2}$ on $\partial V$.

Let $K \subset$ int $V$ be the $(p, q)$-cable of $K^{\prime}$, the core of $V$. Then $X=M-$ int $N(K)=X_{0} \cup(V-$ int $N(K))=X_{0} \cup C$, where $C$ is a $(p, q)$-cable space (see [13], §3). There exists a planar surface $P \subset C$ with one boundary component on $\partial V$, with slope $(1+k p q) / k q^{2}$, and $q$ boundary components on $\partial N(K)$, with slope $(1+k p q) / k$ (see Lemma 3.1 in [13]). Let $S_{0}$ be a Seifert surface for $K_{0}$, and define $S=P \cup S_{0}$, glued along $\partial S_{0}=P \cap \partial V$. Since each of the $q$ components of $S \cap \partial N(K)$ has intersection number $k$ with the meridian of $K$, it follows that $S$ is a $q k$-Seifert surface for $K$. Therefore $\|K\| \leq \eta(S) / q k=\left(\eta\left(S_{0}\right)+(q-1) / 2\right) / q k$, which goes to 0 as $k$ goes to infinity. Also, for $k$ sufficiently large, $M$ is hyperbolic and $K^{\prime}$ is the core of a Margulis tube in $M$.

Case E. ( $M$ is a Seifert fiber space and $K$ is a cable of an exceptional fiber but not a fiber.) Repeat the construction in Case D above, but with $K_{0}$ a $(u, v)$-torus knot. Then $M$ is a Seifert fiber space and $K^{\prime}$ is a fiber of multiplicity $\Delta\left(-k q^{2} / b, u v / 1\right)=$ $\left|k q^{2}+b u v\right|$, which we can arrange to be $>1$. Then $K$ is the $(p, q)$-cable of an exceptional fiber. On the other hand it is easy to see that $K$ can be a fiber in the Seifert fibration of $M$ for at most one value of $k$.

Case F. ( $M$ is toroidal and $K$ lies in a torus in the JSJ decomposition of $M$.) Let $K_{1}, K_{2}$ be non-trivial knots in $S^{3}$, and let $K^{\prime}=K_{1} \# K_{2}$. Let $M$ be the manifold obtained by $m$-surgery on $K^{\prime}$ for some $m>0$, and let $K$ be the core of the surgery solid torus. By Lemma $2.15\|K\|=\left\|K^{\prime}\right\| / m$; thus $\|K\|$ is non-zero but can be made arbitrarily small by taking $m$ sufficiently large.

Let $X_{i}=S^{3}-$ int $N\left(K_{i}\right)$ be the exterior of $K_{i}, i=1,2$; then $X=S^{3}-$ int $N\left(K^{\prime}\right)=X_{1} \cup_{A} X_{2}$ where $A$ is a meridional annulus in $\partial X_{i}, i=1,2$. Let $V$ be the surgery solid torus. Note that the boundary slope of $A$ on $\partial X$ (the meridian of $K^{\prime}$ ) intersects the meridian of $V$ once. It follows that $X_{1} \cup V \cong X_{1}$, and so $M=\left(X_{1} \cup X_{2}\right) \cup V \cong X_{1} \cup_{T} X_{2}$, where $T=\partial X_{1}=\partial X_{2}$. Also $K$, the core of $V$, is isotopic into $T$.

If, for example, we take $K_{1}$ to be hyperbolic and $K_{2}$ to be either hyperbolic or a torus knot, then $T$ is the unique torus in the JSJ decomposition of $M$. Note also that in the second case $K$ is not a Seifert fiber in $X_{2}$. If we take $K_{i}$ to be the $\left(p_{i}, q_{i}\right)$ torus 
knot, $i=1,2$, and $m \neq p_{1} q_{1}+p_{2} q_{2}$, then again $T$ is the unique JSJ torus in $M$, and $K$ is not a Seifert fiber of either $X_{1}$ or $X_{2}$.

Further examples are given in the next subsection.

2.5. Torus bundles. In this section we analyze the case where $M$ is a $T^{2}$-bundle over $S^{1}$ and $K$ is an essential simple closed curve in a fiber. It turns out that this gives further examples of knots with arbitrarily small rational genus, which need to be taken account of in the statement of Theorem 5.19.

Let $f: T^{2} \rightarrow T^{2}$ be an orientation preserving diffeomorphism, and let $M_{f}$ be the mapping torus of $f$, obtained from $T^{2} \times I$ by identifying $(x, 0)$ with $(f(x), 1)$ for all $x \in T^{2}$. If we choose a basis for $H_{1}\left(T^{2}\right)$, the automorphism $f_{*}$ of $H_{1}\left(T^{2}\right)$ induced by $f$ is represented by a matrix $A_{f} \in \mathrm{SL}(2, \mathbb{Z})$; the diffeomorphism type of $M_{f}$ depends only on the conjugacy class of $A_{f}$ in $\operatorname{GL}(2, \mathbb{Z})$. Let $K$ in $M_{f}$ be an essential simple closed curve in a fiber. If trace $f_{*} \neq 2$ then $\operatorname{det}\left(A_{f}-I\right) \neq 0$, and so every such $K$ has finite order in $H_{1}\left(M_{f}\right)$. If trace $f_{*}=2$ then $A_{f}$ is conjugate in $\operatorname{GL}(2, \mathbb{Z})$ to $\left(\begin{array}{ll}1 & p \\ 0 & 1\end{array}\right)$ for some $p \geq 0$. If $p=0$ then $M_{f}=T^{3}$ and no $K$ has finite order in $H_{1}\left(M_{f}\right)$, so in the sequel we shall always assume that $f_{*} \neq \operatorname{Id}$. If $p \geq 1$ then there is a unique $K$ with finite order $(=p)$ in $H_{1}\left(M_{f}\right)$.

Let $a$ and $b$ be oriented simple closed curves in $T^{2}$ meeting transversely in a single point, such that $K$ is the image in $M_{f}$ of the curve $a \times\{1 / 2\} \subset T^{2} \times I$. Thinking of $T^{2} \times I$ as $(a \times b) \times I=a \times(b \times I)$ shows that $\left(T^{2} \times I, K\right)=\left(S^{1} \times A^{2}, S^{1} \times\right.$ point $\left.\}\right)$, and therefore $T^{2} \times I-$ int $N(K) \cong S^{1} \times P^{2}$, where $P^{2}$ is a pair of pants. Let the boundary components of $P^{2}$ be $B_{0}, B_{1}$ and $C$, where $S^{1} \times B_{i}=T_{i}=T^{2} \times\{i\}$, $i=0,1$, and $S^{1} \times C=\partial N(K)$. Let $b_{0}, b_{1}, c$ be the homology classes of $B_{0}, B_{1}$ and $C$, respectively, oriented so that $\left[\partial P^{2}\right]=b_{0}-b_{1}+c$, and so that $b_{0}$ and $b_{1}$ map to the class $b$ above in $H_{1}\left(T^{2} \times I\right)$ (we will abuse notation by not distinguishing between $a, b$ and their classes in $\left.H_{1}\left(T^{2}\right)\right)$.

We now wish to describe certain horizontal surfaces in $S^{1} \times P^{2}$. Consider the homomorphism $H_{1}\left(P^{2}\right) \rightarrow \mathbb{Z}$ defined by $b_{0} \mapsto \ell_{0}, b_{1} \mapsto \ell_{1}$ (so $c \mapsto \ell_{1}-\ell_{0}$ ), where $\ell_{0}$ and $\ell_{1}$ are arbitrary integers. Composing with the Hurewicz map we get a homomorphism $\pi_{1}\left(P^{2}\right) \rightarrow \mathbb{Z}$, which is induced by a map $\pi: P^{2} \rightarrow S^{1}$. Let $m$ be an integer $\geq 1$ and let $\sigma: S^{1} \rightarrow S^{1}$ be the connected covering of degree $m$. Let $F \rightarrow P^{2}$ be the $\mathbb{Z} / m \mathbb{Z}$ covering corresponding to the composition of $\pi_{*}$ with the quotient map $\mathbb{Z} \rightarrow \mathbb{Z} / m \mathbb{Z}$. This covering is the pull-back of $\sigma$ under the map $\pi$, in other words we have a commutative diagram

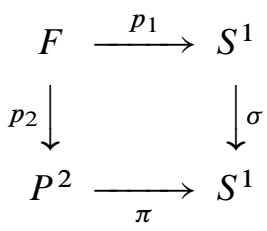


where $F$ is identified with the subset $\{(x, y): \sigma(x)=\pi(y)\}$ of $S^{1} \times P^{2}$, and $p_{1}$ and $p_{2}$ are the restrictions of the projections of $S^{1} \times P^{2}$ onto the factors.

Consider the ordered bases $\left(a, b_{0}\right),\left(a, b_{1}\right)$ and $(a, c)$ for $H_{1}\left(T_{0}\right), H_{1}\left(T_{1}\right)$ and $H_{1}(\partial N(K))$, respectively. Let $\tilde{B}_{0}, \tilde{B}_{1}, \tilde{C}$ be the respective inverse images in $\partial F$ of $B_{0}, B_{1}$ and $C$. Since the coverings $\tilde{B}_{i} \rightarrow B_{i}, i=0,1$, and $\tilde{C} \rightarrow C$ have degree $m$, we have

$$
\begin{aligned}
{\left[\tilde{B}_{i}\right] } & =\ell_{i} a+m b_{i}, \quad i=0,1, \\
{[\tilde{C}] } & =\ell a+m c \quad \text { where } \ell=\ell_{1}-\ell_{0} .
\end{aligned}
$$

In particular, if $\ell \neq 0$ then $F$ is a relative $|\ell|$-Seifert surface for $K$ in $T^{2} \times I$.

Let $f_{*}$ be represented with respect to the basis $(a, b)$ by $\left(\begin{array}{ll}\alpha & \beta \\ \gamma & \delta\end{array}\right) \in \operatorname{SL}(2, \mathbb{Z})$. Assume for the moment that we are not in the case $\gamma=0, \alpha=\delta=-1$. Then we can choose $\ell_{0}$ and $m \geq 1$ such that

$$
\gamma \ell_{0}+(\delta-1) m=0
$$

and define

$$
\ell_{1}=\alpha \ell_{0}+\beta m
$$

so

$$
\ell=(\alpha-1) \ell_{0}+\beta m \text {. }
$$

Then $\left(\begin{array}{ll}\alpha & \beta \\ \gamma & \delta\end{array}\right)\left(\begin{array}{l}\ell_{0} \\ m\end{array}\right)=\left(\begin{array}{l}\ell_{1} \\ m\end{array}\right)$, which implies that we may isotope $f$ so that $f\left(\tilde{B}_{0}\right)=$ $\tilde{B}_{1}$, and hence $F$ becomes an orientable surface $S$ in $M_{f}$. If trace $f_{*} \neq 2$ then (2.5.2) and (2.5.4) imply that $\ell \neq 0$, and so $S$ is an $|\ell|$-Seifert surface for $K$. Since $\chi(S)=\chi(F)=m \chi\left(P^{2}\right)=-m$, we get

$$
\|K\| \leq m / 2|\ell|
$$

We note that if trace $f_{*} \neq 2$ and $\gamma \neq 0$, it follows easily from (2.5.2) and (2.5.4) that

$$
m / \ell=\gamma /\left(\text { trace } f_{*}-2\right) \text {. }
$$

In the case trace $f_{*}=2, A_{f}$ is conjugate to $\left(\begin{array}{ll}1 & p \\ 0 & 1\end{array}\right), p \geq 1$, where the first element of the corresponding ordered basis for $H_{1}\left(T^{2}\right)$ is represented by the unique $K$ that has finite order in $H_{1}\left(M_{f}\right)$. Thus $\left(\begin{array}{ll}\alpha & \beta \\ \gamma & \delta\end{array}\right)=\left(\begin{array}{ll}1 & p \\ 0 & 1\end{array}\right)$, and in (2.5.2) we choose $\ell_{0}=0$, $m=1$, giving $\ell=\ell_{1}=p$.

The above discussion shows that unless $\gamma=0$ and trace $f_{*}=-2$, any $K$ in a fiber of $M_{f}$ having finite order in $H_{1}\left(M_{f}\right)$ has an $|\ell|$-Seifert surface, $\ell \neq 0$, such that the corresponding surface $F$ in $T^{2} \times I-$ int $N(K)=S^{1} \times P^{2}$ is horizontal (i.e. transverse to the $S^{1}$ fibers), and (2.5.5) holds.

It remains to discuss the case $\gamma=0$, trace $f_{*}=-2$, i.e. where our matrix is $\left(\begin{array}{cc}-1 & \beta \\ 0 & -1\end{array}\right)$. Let $A_{i}$ be a vertical annulus in $S^{1} \times P^{2}$ with one boundary component on 
each of $T_{i}$ and $\partial N(K), i=0,1$. Since $f_{*}(a)=-a, A_{0}$ and $A_{1}$ glue up to give an annulus $A$ in $M_{f}$ - int $N(K)$ whose boundary components are coherently oriented on $\partial N(K)$. Hence $\|K\|=0$.

Finally, we show that the inequality (2.5.5) is an equality. Let $S$ be a good $p$ Seifert surface for $K$ in $M_{f}$ such that $\|K\|=\eta(S) / p$. Let $T \subset M_{f}$ be the fiber that is the image of $T^{2} \times \partial I$; note that $K \cap T=\emptyset$. Isotoping $S$ to minimize the number of components of $S \cap T$ we get a relative $p$-Seifert surface $F$ for $K$ in $T^{2} \times I$ that is essential in $T^{2} \times I-\operatorname{int} N(K)=S^{1} \times P^{2}$. Therefore $F$ is either horizontal or vertical.

First we dispose of the vertical case. Here $F$ must consist of either a single annulus with both boundary components on $\partial N(K)$, or two annuli, one running from $\partial N(K)$ to $T_{0}$ and the other from $\partial N(K)$ to $T_{1}$. In the first case, $[\partial S]=[\partial F]=0 \in H_{1}(\partial N(K))$, a contradiction. In the second case, we also get $[\partial S]=0 \in H_{1}(\partial N(K))$ unless $f_{*}(a)=-a$, in which case $A_{0}$ and $A_{1}$ glue up to give an annulus $A$ in $M_{f}-$ int $N(K)$ that is a 2-Seifert surface for $K$, implying that $\|K\|=0$. This is precisely the case $\gamma=0$, trace $f_{*}=-2$ discussed above.

Now suppose $F$ is horizontal. Then the restriction to $F$ of $p_{2}: S^{1} \times P^{2} \rightarrow P^{2}$ is a covering projection, of degree $m \geq 1$, say. Then, with the same notation as used earlier, we see that (2.5.1) must hold, and the subsequent discussion shows that (2.5.2) holds for some $\ell_{0}$, that $p=|\ell|$ where $\ell=\ell_{1}-\ell_{0}$, and thence that $\|K\|=\eta(S) /|\ell|=m / 2|\ell|$.

The following theorem summarizes our conclusions.

Theorem 2.21. Let $M_{f}$ be a $T^{2}$-bundle over $S^{1}$ with monodromy $f$ not isotopic to the identity, and let $K$ be an essential simple closed curve in a fiber.

(1) If trace $f_{*}=2$ then there is a unique $K$ that has finite order in $H_{1}\left(M_{f}\right)$, and $\|K\|=1 / 2 p$ where $f_{*}$ is represented by the matrix $\left(\begin{array}{ll}1 & p \\ 0 & 1\end{array}\right), p \geq 1$.

(2) If trace $f_{*} \neq 2$ then every $K$ has finite order in $H_{1}\left(M_{f}\right)$, and

$$
\|K\|=\mid \gamma / 2\left(\text { trace } f_{*}-2\right) \mid
$$

where $f_{*}$ is represented by the matrix $\left(\begin{array}{ll}\alpha & \beta \\ \gamma & \delta\end{array}\right)$ with respect to an ordered basis of $H_{1}\left(T^{2}\right)$ whose first member is $[K]$.

We see immediately from Theorem 2.21 that knots in fibers of torus bundles provide additional examples of knots with arbitrarily small rational genus. These examples are relevant to Theorem 5.19, cases (4) and (5). We now describe this in more detail, continuing the list in $\$ 2.4$.

Case G. ( $M$ is a Seifert fiber space with no exceptional fibers and $K$ is a fiber.) Let $M$ be $M_{f}$ where trace $f_{*}=2$, as in part (1) of Theorem 2.21. Then $M$ can also be described as an $S^{1}$-bundle over $T^{2}$ with Euler number $p$, and $K$ is a fiber. We remark 
that $M$ has a Nil geometric structure (see [24] pp. 467-470). By taking $p$ large we can make $\|K\|=1 / 2 p$ arbitrarily small.

Case H. ( $M$ is a $T^{2}$-bundle over $S^{1}$ with Anosov monodromy and $K$ lies in a fiber.) Let $M$ be $M_{f}$ with $\mid$ trace $f_{*} \mid>2$, so the monodromy $f$ is Anosov. Note that $M$ has a Sol geometric structure (see [24], pp. 470-472). It is clear from the formula in part (2) of Theorem 2.21 that we can choose $M$ and $K$ so that $\|K\|$ is arbitrarily small. For example, choose any matrix $\left(\begin{array}{ll}\alpha & \beta \\ \gamma & \delta\end{array}\right) \in \operatorname{SL}(2, \mathbb{Z})$ with $\gamma \neq 0$ and let $f_{n}: T^{2} \rightarrow T^{2}$ be given by the matrix $\left(\begin{array}{ll}1 & n \\ 0 & 1\end{array}\right)\left(\begin{array}{ll}\alpha & \beta \\ \gamma & \delta\end{array}\right)=\left(\begin{array}{cc}\alpha+n \gamma & \beta+n \delta \\ \gamma & \delta\end{array}\right)$ with respect to some basis $(a, b)$, say. Let $M_{n}$ be the corresponding $T^{2}$-bundle and let $K_{n}$ be the knot in a fiber such that $\left[K_{n}\right]=a$. Then $\left\|K_{n}\right\|=|\gamma / 2(\alpha+\delta+n \gamma-2)| \rightarrow 0$ as $n \rightarrow \infty$.

Remark 2.22. It follows from Theorem 2.21 that if we are not in Case $\mathrm{G}$ or Case $\mathrm{H}$, i.e. if trace $f_{*}=-2$ or $\mid$ trace $f_{*} \mid \leq 1$, then either $\|K\|=0$ or $\|K\| \geq 1 / 8$.

2.6. Knots in vertical tori in Seifert fiber spaces. In this section we analyze relative $p$-Seifert surfaces for knots that lie in essential vertical tori in Seifert fiber spaces. First we have the following lemma.

Lemma 2.23. Let $M$ be a Seifert fiber space with non-empty boundary and let $\pi: M \rightarrow \mathcal{B}$ be the projection of $M$ onto its base orbifold $\mathcal{B}$. Let $F$ be a horizontal surface in $M$ and let $k$ be the degree of the induced branched covering $\pi \mid F: F \rightarrow B$. If $\chi(F)<0$ then $\chi(F) \leq-k / 6$.

Proof. Let $q_{1}, \ldots, q_{n}$ be the multiplicities of the exceptional fibers of $M$. Then (see for example [14], §2.1)

$$
\chi(F)=k\left(\chi(\mathcal{B})-\sum_{i=1}^{n}\left(1-\frac{1}{q_{i}}\right)\right) .
$$

Since $\chi(F)<0$, the maximal value of the expression in parentheses is attained when $\chi(\mathscr{B})=1, n=2, q_{1}=2, q_{2}=3$, which gives the value $-1 / 6$.

Proposition 2.24. Let $M$ be a Seifert fiber space, $T$ a vertical essential torus in $M$, $K$ an essential simple closed curve in $T$, and $F$ a relative $p$-Seifert surface for $K$. Then either

(1) $-\chi^{-}(F) \geq p / 6$; or

(2) $K$ is an ordinary fiber in the Seifert fibration of $M$; or

(3) $M$ contains a submanifold $Q$ that is a twisted $S^{1}$-bundle over the Möbius band and $K$ is a fiber in $Q$. 
Note that if (3) holds but (2) does not then the Seifert fibration of $Q$ induced from $M$ is the one with base orbifold a disk with two cone points of order 2 .

Proof. Let $T^{2} \times I$ be a regular neighborhood of $T=T^{2} \times\{1 / 2\}$. First suppose that $T$ separates $M$. Then $M=X_{0} \cup T^{2} \times I \cup X_{1}$, where $T_{i}=T^{2} \times\{i\}$ is a component of $\partial X_{i}, i=0,1$. Let $N(K)$ be a regular neighborhood of $K$ in $T^{2} \times I$ and let $Y=T^{2} \times I-$ int $N(K)$. Then $X=M-$ int $N(K)=X_{0} \cup Y \cup X_{1}$. Let $F$ be a good relative $p$-Seifert surface for $K$ in $M$. Let $F_{i}=F \cap X_{i}, i=0,1$, and $G=F \cap Y$. We may assume that $F_{0}, F_{1}$ and $G$ are essential in $X_{0}, X_{1}$ and $Y$ respectively. The Seifert fibration of $M$ induces Seifert fiber structures on $X_{0}$ and $X_{1}$, with base orbifolds $\mathcal{B}_{0}$ and $\mathcal{B}_{1}$, say. Recall from $\S 2.5$ that $Y \cong S^{1} \times P^{2}$ where $P^{2}$ is a pair of pants.

Note that $F_{i}$ is horizontal or vertical in $X_{i}, i=0,1$, and $G$ is horizontal or vertical in $Y \cong S^{1} \times P^{2}$. Write $\partial_{i} F_{i}=\partial F_{i} \cap T_{i}=\partial G \cap T_{i}=\partial_{i} G, i=0,1$.

Case I. $G$ is vertical. Since a vertical annulus in $Y$ that has both its boundary components on $\partial N(K)$ has these boundary components oriented oppositely on $\partial N(K)$, and since $F$ is good, it follows that $G$ consists of $p$ parallel copies of an annulus with one boundary component on $\partial N(K)$ and the other on (say) $T_{0}, F_{1}=\emptyset$, and $F_{0}$ is connected.

Subcase (a). $\chi\left(F_{0}\right)<0$. Then $F_{0}$ is horizontal in $X_{0}$. Since $\partial_{0} F_{0}$ has $p$ components the index of the covering $F_{0} \rightarrow \mathscr{B}_{0}$ is at least $p$. Therefore, by Lemma 2.23, $\chi(F)=\chi\left(F_{0}\right) \leq-p / 6$.

Subcase (b). $\chi\left(F_{0}\right)=0$. Then $F_{0}$ is an annulus. First suppose that $\partial_{0} F_{0}$ has a single component. If $F_{0}$ is horizontal then $X_{0} \cong F_{0} \times S^{1} \cong T_{0} \times I$, contradicting the assumption that $T$ is essential in $M$. If $F_{0}$ is vertical then $K$ is an ordinary fiber in the Seifert fibration of $M$.

If $\partial_{0} F_{0}$ has two components then $F$ is an annulus with both boundary components on $\partial N(K)$ and by Theorem $2.11 K$ is contained in a submanifold $N$ of $M$ where $N$ is a Seifert fiber space over the Möbius band with at most one orbifold point of order $r \geq 1$ and $K$ is a fiber of multiplicity $r$. If $r>1$ then, since the Seifert fibration of $N$ is unique, $K$ is an exceptional fiber in $M$. But this contradicts the fact that $K$ is contained in a vertical torus. Hence $r=1$ and we have conclusion (3).

This completes the proof in Case I.

Case II. $G$ is horizontal. Here we will adopt the notation of $\S 2.5$. Let $m$ be the index of the covering $G \rightarrow P^{2}$; so $\chi(G)=-m$. Also we have

$$
\begin{gathered}
{\left[\partial_{i} F_{i}\right]=\left[\partial_{i} G\right]=\ell_{i} a+m b_{i}, \quad i=0,1,} \\
{[\partial G \cap N(K)]=\left(\ell_{1}-\ell_{0}\right) a+m c,}
\end{gathered}
$$

where $p=\left|\ell_{1}-\ell_{0}\right|$. 
Let $\varphi_{i}$ be the Seifert fiber of $M$ on $T_{i}, i=0,1$. Then $\left[\varphi_{i}\right]=\alpha a+\beta b_{i}$, say, $i=0,1$. If $\beta=0$ then $[K]=\left[\varphi_{i}\right]$ so $K$ is an ordinary fiber in the Seifert fibration of $M$. We will therefore assume that $\beta \neq 0$.

If $F_{i}$ is horizontal in $X_{i}$, let $k_{i}$ denote the index of the associated covering $F_{i} \rightarrow$ $\mathscr{B}_{i}, i=0,1$. Then $k_{i}=\left|\partial_{i} F_{i} \cdot \varphi_{i}\right|=\left|\left(\ell_{i} a+m b_{i}\right) \cdot\left(\alpha a+\beta b_{i}\right)\right|=\left|\beta \ell_{i}-\alpha m\right|$, where $\cdot$ denotes algebraic intersection number.

Sublemma 2.25. (1) If both $F_{0}$ and $F_{1}$ are horizontal then $k_{0}+k_{1} \geq p$.

(2) If $F_{i}$ is horizontal and $\chi\left(F_{i}\right)=0$ then $k_{i} \leq m$.

(3) If $F_{0}$ is horizontal and $F_{1}$ is vertical then $k_{0} \geq p$.

Proof. (1) $k_{0}+k_{1}=\left|\beta \ell_{0}-\alpha m\right|+\left|\beta \ell_{1}-\alpha m\right| \geq|\beta|\left|\ell_{1}-\ell_{0}\right|=|\beta| p \geq p$.

(2) Here $F_{i}$ consists of parallel copies of a horizontal annulus $A$ in $X_{i}$. If $A$ has one boundary component on $T_{i}$ and one on $\partial M$ then $X_{i} \cong A \times S^{1} \cong T_{i} \times I$, contradicting the assumption that $T$ is essential in $M$. Also, since the components of $\partial_{i} G$ are coherently oriented on $T_{i}$ the same holds for $\partial_{i} F_{i}$. It follows that $A$ is non-separating and $\mathscr{B}_{i}$ is a disk with two cone points of order 2. In particular each boundary component of $A$ has intersection number 1 with the Seifert fiber $\varphi_{i}$. Therefore $k_{i}=\left|\partial_{i} F_{i} \cdot \varphi_{i}\right|$ is the number of components of $\partial_{i} F_{i}=\partial_{i} G$, which is $\leq m$ since $G$ is an $m$-fold covering of $P^{2}$.

(3) If $F_{1}$ is vertical then $\left[\partial_{1} F_{1}\right]=\ell_{1} a+m b_{1}=s\left[\varphi_{1}\right]=s\left(\alpha a+\beta b_{1}\right)$ where $s$ is the number of components of $\partial_{1} F_{1}=\partial_{1} G$. Hence $s \leq m$. Now

$$
\begin{aligned}
s k_{0} & =\left|\partial_{0} F_{0} \cdot s \varphi_{0}\right| \\
& =\left(\ell_{0} a+m b_{0}\right) \cdot\left(\ell_{1} a+m b_{0}\right) \\
& =m\left|\ell_{1}-\ell_{0}\right|=m p .
\end{aligned}
$$

Therefore $k_{0}=m p / s \geq p$.

We now complete the proof of Proposition 2.24 in Case II.

First note that $F_{0}$ and $F_{1}$ cannot both be vertical, for then we would have $\ell_{0}=\ell_{1}$ and hence $p=0$.

Subcase (a). $F_{0}$ and $F_{1}$ horizontal. If $\chi\left(F_{0}\right), \chi\left(F_{1}\right)<0$ then

$$
\begin{aligned}
|\chi(F)| & =\left|\chi\left(F_{0}\right)\right|+\left|\chi\left(F_{1}\right)\right|+|\chi(G)| \\
& \geq\left(k_{0}+k_{1}\right) / 6+m, \text { by Lemma } 2.23 \\
& \geq p / 6+m, \text { by Sublemma } 2.25(1) \\
& >p / 6 .
\end{aligned}
$$


If $\chi\left(F_{0}\right)=0$ and $\chi\left(F_{1}\right)<0$ then $k_{0} \leq m$ by Sublemma 2.25 (2), and hence $k_{1} \geq p-m$ by Sublemma 2.25 (1). Therefore

$$
\begin{aligned}
|\chi(F)| & =\left|\chi\left(F_{1}\right)\right|+|\chi(G)| \\
& \geq k_{1} / 6+m \\
& \geq(p-m) / 6+m>p / 6 .
\end{aligned}
$$

Finally, if $\chi\left(F_{0}\right)=\chi\left(F_{1}\right)=0$, then by Sublemma 2.25 parts (1) and (2), we have $p \leq k_{0}+k_{1} \leq 2 m$. Hence

$$
|\chi(F)|=|\chi(G)|=m \geq p / 2 .
$$

Subcase (b). $F_{0}$ horizontal, $F_{1}$ vertical. If $\chi\left(F_{0}\right)<0$ then

$$
\begin{aligned}
|\chi(F)| & =\left|\chi\left(F_{0}\right)\right|+|\chi(G)| \\
& \geq k_{0} / 6+m \\
& \geq p / 6+m, \text { by Sublemma } 2.25(3) \\
& >p / 6 .
\end{aligned}
$$

If $\chi\left(F_{0}\right)=0$ then by Sublemma 2.25 (2) $k_{0} \leq m$, while by Sublemma 2.25 (3) $k_{0} \geq p$. Hence $|\chi(F)|=|\chi(G)|=m \geq p$.

This completes the proof of Proposition 2.24 when $T$ separates $M$.

If $T$ is non-separating, let $\pi: M \rightarrow \mathscr{B}$ be the projection from $M$ to its base orbifold $\mathscr{B}$. Let $N$ be a regular neighborhood of either the union of the exceptional fibers of $M$ or, if $M$ is closed and has no exceptional fibers, an ordinary fiber. Let $M_{0}=M-$ int $N$, with corresponding base orbifold $\mathscr{B}_{0}$. Then $M_{0}$ is an $S^{1}$-bundle over $\mathscr{B}_{0}$ and $T=\pi^{-1}(C)$ for some non-separating orientation-preserving simple closed curve $C$ in $\mathscr{B}_{0}$. Now $H_{1}(T)$ has basis $\varphi, \gamma$, where $\varphi$ is the class of the $S^{1}$-fiber of $M_{0}$ and $\pi_{*}(\gamma)=[C] \in H_{1}\left(\mathcal{B}_{0}\right)$. Therefore $[K]=r \varphi+s \gamma$ for some pair of relatively prime integers $r, s$.

Isotoping $F$ to be transverse to the core of the components of $N$, let $F_{0}=F \cap M_{0}$. Then $F_{0}$ defines a homology of $p K$ into $\partial M_{0}$. Therefore, considering the map $\pi_{*}: H_{1}\left(M_{0}, \partial M_{0}\right) \rightarrow H_{1}\left(\mathscr{B}_{0}, \partial \mathscr{B}_{0}\right)$, we have $0=\pi_{*}(p[K])=p s[C]$. Since $C$ is orientation-preserving and non-separating, $[C]$ has infinite order in $H_{1}\left(\mathscr{B}_{0}, \partial \mathscr{B}_{0}\right)$, and so we conclude that $s=0$. Therefore $K$ is an ordinary fiber in the Seifert fibration of $M$.

\section{Graphs}

Several of our arguments concern the interaction between a relative $p$-Seifert surface $F$ for a knot $K$ in $M$, and another surface $\widehat{G}$ properly embedded in $M$. Such 
arguments are handled in a uniform manner, by making the surfaces intersect as simply as possible, and then by analyzing cases depending on the combinatorics of this intersection. The arguments in this section are mostly combinatorial.

3.1. Graphs on surfaces. Fix the following notation. Let $K$ denote a knot in $M$, let $F$ be a good relative $p$-Seifert surface for $K$, and let $\widehat{G}$ be a properly embedded surface in $M$ (usually a Heegaard surface, or an essential surface; usually of low complexity). Under such a circumstance, we perform the following procedure.

Isotop $N(K)$ so that it meets $\widehat{G}$ in $n$ meridian disks, and let $G=\widehat{G} \cap X$, so that $F$ and $G$ are both proper surfaces in $X$. After an isotopy, we may assume that $F$ and $G$ meet transversely in a finite disjoint union of circles and properly embedded arcs and that each of the $q$ components of $\partial F \cap \partial N(K)$ meets each of the $n$ components of $\partial G \cap \partial N(K)$ in $r$ points, with notation as in Definition 2.5.

Formally cap off the components of $\partial F \cap \partial N(K)$ with disks to obtain a surface $\widehat{F}$ (note: if $F$ is an honest $p$-Seifert surface, then $\widehat{F}$ is closed. Otherwise, $\partial \widehat{F}=$ $\partial F \cap \partial M)$. The intersection $F \cap G$ determines graphs $\Gamma_{F}$ and $\Gamma_{G}$ in $\widehat{F}$ and $\widehat{G}$ respectively, where the vertices of $\Gamma_{F}$ (resp. the vertices of $\Gamma_{G}$ ) correspond to the disks of $\hat{F}-F$ (resp. the disks of $\widehat{G}-G$ ) and the edges correspond to the arc components of $F \cap G$ with at least one endpoint on $\partial N(K)$. We distinguish between two kinds of edges of $\Gamma_{F}$ and $\Gamma_{G}$ : interior edges, which have both endpoints on $\partial N(K)$ (i.e. at the vertices), and boundary edges, which have one endpoint on $\partial N(K)$, and the other on $\partial \widehat{F}=F \cap \partial M$ or $\partial \widehat{G}=G \cap \partial M$.

Choose orientations on $F, G$ and $X$. This induces orientations on $\partial F, \partial G$ and $\partial X$, and an arc of $F \cap G$ joins points of intersection of $\partial F$ with $\partial G$ of opposite sign.

Number the components of $\partial G \cap \partial N(K)$ (equivalently, the vertices of $\Gamma_{G}$ ) with the integers $1,2, \ldots, n$ in the (cyclic) order they occur along $\partial N(K)$. Hereafter an index means an element of this index set; i.e. an element $i \in\{1, \ldots, n\}$. We imagine that the vertices of $\Gamma_{F}$ and $\Gamma_{G}$ are thickened, so that distinct edges end at distinct "edge-endpoints" on a (thickened) vertex. With this convention, an edgeendpoint at a vertex of $\Gamma_{F}$ is a point of intersection of the corresponding component of $\partial F \cap \partial N(K)$ with a component of $\partial G \cap \partial N(K)$, and we label the edge-endpoint with the index corresponding to the label on the component of $\partial G \cap \partial N(K)$. Notice that it is the vertices of $\Gamma_{G}$ and the edge-endpoints of $\Gamma_{F}$ that are labeled with indices. Since $F$ is good (by hypothesis), all components of $\partial F \cap \partial N(K)$ are coherently oriented on $\partial N(K)$, and therefore at each vertex of $\Gamma_{F}$ we see the index labels $1,2, \ldots, n, 1,2, \ldots, n, \ldots$ repeated $r$ times in (say) anticlockwise order on the edgeendpoints around the vertex. Notice that if $\Gamma_{F}$ and $\Gamma_{G}$ have $e_{i}$ interior edges and $e_{\partial}$ boundary edges, then $2 e_{i}+e_{\partial}=p n$.

In applications, the surface $\widehat{G}$ will always be either essential, or a Heegaard surface. In the former case we will choose $n=|K \cap \widehat{G}|$ to be minimal; and in the latter case we will put $K$ in thin position. 
Remark 3.1. Thin position for knots in $S^{3}$ was introduced by Gabai [12], and for knots in arbitrary 3-manifolds by Rieck [20], [21], and we refer to these references for details. Technically, a knot is in thin position with respect to a sweepout of a 3 -manifold (associated to a Heegaard splitting). The Heegaard surface $\widehat{G}$ is one of the nonsingular level sets of this sweepout, chosen depending on $F$.

Lemma 3.2. With notation and conventions as above, we can arrange that no arc component of $F \cap G$ with both endpoints in $\partial N(K)$ is boundary parallel in either $F$ or $G$. Equivalently, the graphs $\Gamma_{F}$ and $\Gamma_{G}$ have no monogon (disk) faces.

Proof. The arguments are standard. Since components of $\partial F$ are oriented coherently on $\partial N(K)$, every point of intersection of a given component of $\partial G$ with $\partial F$ has the same sign. Hence in particular, every interior edge of $\Gamma_{G}$ has endpoints on distinct vertices of $\Gamma_{G}$, and there are never any complementary monogons.

If there is a monogon complementary to $\Gamma_{F}$ then $\widehat{G}$ can be pushed over such a monogon by an isotopy, thereby reducing the number of intersections with $K$; this is ruled out by hypothesis when $\widehat{G}$ is essential.

It remains to rule out monogon regions for $\Gamma_{F}$ when $\widehat{G}$ is a Heegaard surface (note that such monogons may contain interior loops of $F \cap \widehat{G}$ that bound compressing disks for $\widehat{G}$; see footnote 12 on page 635 of [21]). Such a monogon region is either a high disk or a low disk for $\widehat{G}$, in the terminology of [21]. The existence of disjoint high and low disks at some level violates thinness; Gabai's argument in [12] (also see Theorem 6.2 in [21]) shows that for a knot in thin position, some level set of the sweepout admits neither. Choosing $\widehat{G}$ to be such a level set, $\Gamma_{F}$ has no monogons.

Remark 3.3. If $F \cap G$ has a simple closed curve component that bounds a disk in $G$, let $\gamma$ be an innermost such, i.e. $\gamma$ bounds a disk $D$ in $G$ such that $F \cap($ int $D)=\emptyset$. Since $F$ is incompressible in $X$, the loop $\gamma$ bounds a disk $E$ in $F$. Surgering $F$ along $D$ produces a 2-sphere $\Sigma=E \cup D$ together with a surface $F^{\prime}$ that is essential in $X$, has $\partial F^{\prime}=\partial F$, and is homeomorphic to $F$, and which may be isotoped so that $\left|F^{\prime} \cap G\right|<|F \cap G|$. If $M$ is irreducible then $\Sigma$ bounds a 3-ball and $F^{\prime}$ is isotopic to $F$. So in that case we may assume that no simple closed curve component of $F \cap G$ bounds a disk in $G$.

If $\widehat{G}$ is essential we will always assume that $n=|K \cap \widehat{G}|$ is minimal over all essential surfaces $\widehat{G}$ in $M$ of the given homeomorphism type. This implies that $G$ is essential in $X$. Hence by the remarks above, interchanging the roles of $F$ and $G$, we may assume that no simple closed curve component of $F \cap G$ bounds a disk in $F$.

Let $\Gamma_{F}$ as above be a graph on $\widehat{F}$ without monogons. If every complementary region to $\Gamma_{F}$ is a bigon, then either $\widehat{F}$ is a sphere, $\Gamma_{F}$ has exactly two vertices, with parallel interior edges running between them, or $\hat{F}$ is a disk, $\Gamma_{F}$ has exactly one 
vertex, with parallel boundary edges running from the vertex to the boundary. We call such a $\Gamma_{F}$ a beachball (Figure 1 indicates why), of the first kind and second kind respectively.

Remark 3.4. If $\Gamma_{F}$ is a beachball of the first kind then $\|K\|=0$ and $K$ satisfies conclusion (3) of Theorem 2.11.

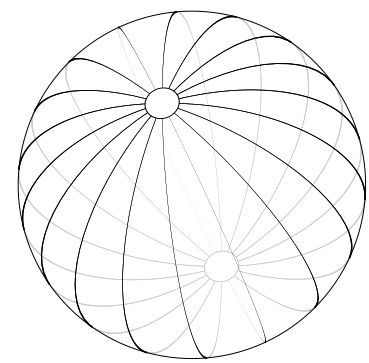

Figure 1. A beachball of the first kind, with 18 complementary bigon regions.

Suppose $\Gamma_{F}$ is not a beachball. The reduced graph $\bar{\Gamma}_{F}$ associated to $\Gamma_{F}$ is obtained from $\Gamma_{F}$ by collapsing all bigon regions. More generally, a reduced graph $\bar{\Gamma}$ in a surface $\widehat{F}$ is any graph with no complementary monogon or bigon regions.

Lemma 3.5. Let $\bar{\Gamma}$ be a reduced graph in $\widehat{F}$ with $\bar{e}$ edges. Then $-\chi(F) \geq \bar{e} / 3$.

Proof. Let $\bar{v}$ be the number of vertices of $\bar{\Gamma}$, and $\bar{f}$ the number of complementary disk faces. Non-disk faces contribute non-positively to Euler characteristic, so $\chi(\widehat{F}) \leq$ $\bar{v}-\bar{e}+\bar{f}$. Hence $\chi(F)=\chi(\widehat{F})-\bar{v} \leq \bar{f}-\bar{e}$. Since $\bar{\Gamma}$ is reduced, it has no monogon or bigon faces, so $2 \bar{e} \geq 3 \bar{f}$. Hence $3 \chi(F) \leq 3 \bar{f}-3 \bar{e} \leq-\bar{e}$.

Edges in $\Gamma_{F}$ that cobound a bigon are said to be parallel. If $\Gamma_{F}$ is complicated, either $-\chi(F)$ is large by Lemma 3.5, or else there are many parallel edges. The next lemma discusses the latter possibility. But first we introduce some terminology.

Notation 3.6. An interior edge of $\Gamma_{G}$ that joins vertices with index labels $i$ and $j$ will be called an $(i, j)$-edge.

Lemma 3.7. If $\Gamma_{F}$ contains $(m n+1)$ parallel interior edges where $m \geq 1$, then there exists an index $k$ such that

(1) for each index $i$, the graph $\Gamma_{G}$ has $2 m$ edges which are ( $\left.i, k-i\right)$-edges; and

(2) for some index $i_{0}$, the graph $\Gamma_{G}$ has $(2 m+1)$ edges which are $\left(i_{0}, k-i_{0}\right)$-edges. 
Proof. Since all boundary components of $F$ are oriented coherently on $K$ (one says the vertices of $\Gamma_{F}$ have the same sign), there is some (odd) index $k$ such that the index labels of any edge in the family are $i$ and $k-i(\operatorname{taken} \bmod n)$. Since the family contains $(m n+1)$ edges, there is an index label $i_{0}$ that appears $(m+1)$ times at one end of the family, and $m$ times at the other end; this proves the second claim. Moreover, any index label $i$ appears at least $m$ times at each end, proving the first claim.

Every edge of $\Gamma_{G}$ is an arc of intersection of $F$ with $G$, and therefore corresponds to an edge of $\Gamma_{F}$, and conversely. The next two lemmas control what happens when there are pairs of edges that are parallel on both graphs simultaneously.

Lemma 3.8. Suppose there are interior edges that are parallel on both $\Gamma_{F}$ and $\Gamma_{G}$. Then $(M, K)=\left(M^{\prime}, K^{\prime}\right) \#\left(\mathbb{R} \mathbb{P}^{3}, \mathbb{R} \mathbb{P}^{1}\right)$.

Proof. Since all vertices of $\Gamma_{F}$ have the same sign, this follows from the argument in [13], proof of Proposition 1.3. We observe that this argument is still valid if $F \cap G$ has simple closed curve components.

Lemma 3.9. Suppose there are boundary edges that are parallel on both $\Gamma_{F}$ and $\Gamma_{G}$. Then $K$ is isotopic into $\partial M$.

Proof. By [11], Lemma 2.5.4, such a pair of boundary edges gives an essential annulus $A$ in $X$ with one boundary component on $\partial M$ and one on $\partial N(K)$, the latter having intersection number 1 with the meridian of $K$. Again this argument is valid in the presence of simple closed curve components of $F \cap G$. This annulus can be used to define an isotopy of $K$ into $\partial M$.

3.2. Cables, satellites and tori. In the sequel, many arguments will depend on relativizing to a knot in a simple 3-manifold with boundary (i.e. a submanifold of $M)$. In this section, we analyze the most important special cases.

Definition 3.10. Let $K$ be a knot in $M$, with regular neighborhood $N(K)$. Let $K^{\prime}$ be a simple closed curve on $\partial N(K)$ that is essential in $N(K)$. Then we call $K^{\prime}$ a cable of $K$.

A knot $K^{\prime}$ contained in $N(K)$ is called a satellite of $K$ if it is not contained in a 3-ball in $N(K)$. If $\left[K^{\prime}\right]=k[K] \in H_{1}(N(K))$ then $k$ is called the winding number of the satellite.

Remark 3.11. Note that our definitions of a satellite and of a cable include the trivial cases where $K^{\prime}$ is isotopic to $K$ in $N(K)$. 
Proposition 3.12. Let $K_{0}$ be a knot in a 3-manifold $M$ whose exterior has incompressible boundary, and let $K$ be a satellite of $K_{0}$ with winding number $k>0$. Then $\|K\| \geq k\left\|K_{0}\right\|$.

Proof. By the definition of satellite, $K$ is contained in a solid torus $V$ in $M$ whose core is $K_{0}$. Let $X_{0}=M-$ int $V$. Let $S$ be a good $p$-Seifert surface for $K$, $F=S \cap(V-$ int $N(K))$, and $S_{0}=S \cap X_{0}$.

If $K_{0}$ is $p$-trivial for some $p$ the result is obvious. So we may assume that $\partial V$ is incompressible in $X_{0}$ (see Remark 2.16). Thus $\partial V$ is incompressible in $M-K$, and we may therefore assume that no component of $S_{0}$ or $F$ is a disk. Hence $\eta(S)=$ $\eta(F)+\eta\left(S_{0}\right)$.

In $H_{1}(V)$, there is equality $\left[\partial S_{0}\right]=[\partial S]=p[K]=p k\left[K_{0}\right]$. Therefore

$$
\left\|K_{0}\right\| \leq \eta\left(S_{0}\right) / p k \leq \eta(S) / p k .
$$

Since $S$ can be chosen so that $\eta(S) / p$ is arbitrarily close to $\|K\|$, the result follows (or one can just apply Proposition 2.10).

Proposition 3.13. Let $M$ be a 3-manifold whose boundary contains a compressible torus $T$, and let $K$ be a knot in $M$ such that $T$ is incompressible in $M-K$. Let $F$ be a relative $p$-Seifert surface for $K$. Then either

(1) $-\chi^{-}(F)>p / 6$; or

(2) $K$ is isotopic into $T$.

Proof. It is enough to prove the proposition under the assumption that $F$ is good.

Let $D$ be a compressing disk for $\partial M$ in $M$, such that $D \cap N(K)$ consists of $n$ meridian disks of $N(K)$, with $n$ minimal. By hypothesis, $n>0$. Let $X=$ $M-$ int $N(K)$ as usual.

Let $P=D \cap X$, a planar surface. By the minimality of $n$, the surface $P$ is incompressible in $X$. Let $\Gamma_{F}$ and $\Gamma_{P}$ be the intersection graphs in $\hat{F}$ and $D$ respectively. There are two cases to consider.

Case A. $(n=1)$ In this case, $P$ is an annulus, $\Gamma_{F}$ and $\Gamma_{P}$ have $p$ edges, and all edges are boundary edges. In particular, all edges of $\Gamma_{P}$ are parallel. If $\Gamma_{F}$ has a pair of parallel edges then $K$ is isotopic into $T$ by the proof of Lemma 3.9. If not, then either $\Gamma_{F}$ is a beachball of the second kind with a single edge, or the reduced graph $\bar{\Gamma}_{F}$ is defined and is equal to $\Gamma_{F}$. In the first case the annulus $F$ defines an isotopy of $K$ into $T$. In the second case $-\chi(F) \geq p / 3$ by Lemma 3.5.

Case B. $(n>1)$ This case depends on two sublemmas.

Sublemma 3.14. The graph $\Gamma_{F}$ contains no family of $(\lfloor n / 2\rfloor+1)$ parallel interior edges. 
Proof. Since all the vertices of $\Gamma_{F}$ have the same sign, such a family would contain a (length 2) Scharlemann cycle (hereafter referred to as an $S$-cycle) i.e. a configuration of the form depicted in Figure 2. As in [22], Proposition 4.7, this $S$-cycle can be

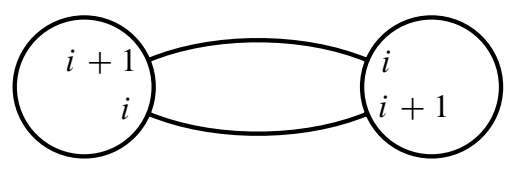

Figure 2. An $S$-cycle.

used to tube and compress $D$, giving a new compressing disk $D^{\prime}$ with $\partial D^{\prime}=\partial D$ and $|D \cap K|=n-2$, contradicting minimality of $n$.

Sublemma 3.15. If $\Gamma_{F}$ contains $(2 n-1)$ parallel boundary edges then $K$ is isotopic into $T$.

Proof. In a family of $(2 n-1)$ parallel boundary edges, the labels (on the vertex at one end of the family) cycle through a full set of labels twice, with exactly one exception. Hence in $\Gamma_{P}$ we get a pair of boundary edges at each vertex except (at most) one. Since $D$ is a disk, a pair of boundary edges together with a common vertex separates $D$, so there is an outermost pair with the property that one of the complementary regions contains no other vertex of $\Gamma_{P}$. But this means that the outermost pair of boundary edges are in fact parallel, so we obtain a pair of boundary edges that are parallel in both $\Gamma_{F}$ and $\Gamma_{P}$. The desired result now follows from the proof of Lemma 3.9.

We now complete the proof in Case B. First note that Sublemma 3.14 implies that $\Gamma_{F}$ is not a beachball of the first kind. If $\Gamma_{F}$ is a beachball of the second kind then by Sublemma 3.15 we may assume that $p=1$. But then the boundary component of $F$ that lies on $\partial N(K)$ intersects the meridian of $K$ exactly once, and so $F$ defines an isotopy of $K$ into $T$. We may therefore suppose that the reduced graph $\bar{\Gamma}_{F}$ of $\Gamma_{F}$ on $\widehat{F}$ exists, with, say, $\bar{e}_{i}$ interior edges and $\bar{e}_{\partial}$ boundary edges. By Sublemma 3.14 each interior edge of $\bar{\Gamma}_{F}$ corresponds to at most $\lfloor n / 2\rfloor$ edges of $\Gamma_{F}$, and by Sublemma 3.15 we may assume that each boundary edge of $\bar{\Gamma}_{F}$ corresponds to at most $(2 n-2)$ edges of $\Gamma_{F}$. Thus edges of $\bar{\Gamma}_{F}$ contribute at most $n$ and $(2 n-2)$ to the sum of valences at all vertices of $\Gamma_{F}$ respectively. Since this total sum is $p n$, and since $n \leq 2 n-2$, we get $\bar{e}=\bar{e}_{i}+\bar{e}_{\partial} \geq p n /(2 n-2)>p / 2$. The conclusion now follows from Lemma 3.5.

Corollary 3.16. Let $K_{0}$ be a knot in a 3-manifold $M$ that is not $m$-trivial for any $m$, and let $K$ be a non-trivial cable of a non-trivial cable of $K_{0}$. Then $\|K\|>1 / 12$.

Proof. Let $V$ be a regular neighborhood of $K_{0}$ containing $K$, and let $X_{0}=M-$ int $V$. By the hypothesis on $K_{0}, \partial V$ is incompressible in $X_{0}$. Let $S$ be a good $p$-Seifert 
surface for $K$ in $M$, such that $\|K\|=\eta(S) / p$, and let $F=S \cap V$ and $S_{0}=S \cap X_{0}$. Then $F$ is a relative $p$-Seifert surface for $K$ in $V$. Since $\partial V$ is incompressible in $M-K$, we may assume that no component of $F$ or $S_{0}$ is a disk, and hence $\eta(S)=\eta(F)+\eta\left(S_{0}\right)$, giving $\eta(F) \leq \eta(S)$.

Since $K$ is not a cable of $K_{0}$, Proposition 3.13, applied to $K \subset V$, implies that $-\chi(F)>p / 6$. Therefore $\|K\|=\eta(S) / p \geq \eta(F) / p>1 / 12$.

The next proposition considers knots contained in a neighborhood of a torus.

Proposition 3.17. Let $K$ be a knot in $T^{2} \times I$, and let $F$ be a relative $p$-Seifert surface for $K$. Then either

(1) $-\chi^{-}(F) \geq p / 3$; or

(2) $K$ is isotopic into $T^{2} \times\{1 / 2\}$.

Proof. We assume $F$ is good.

Put $K$ in thin position with respect to the torus $\widehat{T}:=T^{2} \times\{1 / 2\}$. Let $n=|K \cap \widehat{T}|$, an even integer $\geq 0$. If $n=0$ the second conclusion holds, so assume $n \geq 2$. Construct $\Gamma_{F}, \Gamma_{T}$ without monogons, as in Lemma 3.2. Note that all edges are interior edges. We require a sublemma.

Sublemma 3.18. $\Gamma_{F}$ does not contains a family of $(n / 2+1)$ parallel edges.

Proof. Any such family contains an $S$-cycle. This gives rise to a Möbius band properly embedded in (say) $T^{2} \times[1 / 2,1]$, which is absurd.

By Sublemma 3.18, we obtain an estimate $\bar{e} \geq e /(n / 2)=p$, where $\bar{e}$ is the number of edges in the reduced graph $\bar{\Gamma}_{F}$. (Note that $\bar{\Gamma}_{F}$ exists, since otherwise $\Gamma_{F}$ is a beachball of the first kind and $K$ satisfies part (3) of Theorem 2.11. But this would give a Klein bottle embedded in $T^{2} \times I$, which is absurd.) Now apply Lemma 3.5.

As a corollary, we deduce the following:

Corollary 3.19. Let $T$ be an incompressible torus in a 3-manifold $M$, and let $K$ be a knot in $M$ that lies in a regular neighborhood of $T$. Let $F$ be a relative $p$-Seifert surface for $K$ in $M$. Then either

(1) $-\chi^{-}(F) \geq p / 3$; or

(2) $K$ is isotopic into $T$.

Proof. Let $N$ be a regular neighborhood of $T$, and define $F^{\prime}=F \cap N, F^{\prime \prime}=$ $F \cap(\overline{M-N})$. Since $T$ is incompressible in $M$ we may assume that $F^{\prime \prime}$ has no disk (or sphere) components. Therefore $-\chi^{-}(F) \geq-\chi^{-}\left(F^{\prime}\right)$, and the result follows from Proposition 3.17. 


\section{Hyperbolic knots}

In this section we consider the case that $M-K$ is hyperbolic; i.e. that $K$ is a hyperbolic knot. The arguments in this section use more geometry and analysis.

\subsection{Stable commutator length}

Definition 4.1. Let $G$ be a group, and $a$ an element in $[G, G]$. The commutator length of $a$, denoted by $\operatorname{cl}(a)$, is the minimal number of commutators in $G$ whose product is $a$, and the stable commutator length, denoted by $\operatorname{scl}(a)$, is the limit

$$
\operatorname{scl}(a)=\liminf _{n \rightarrow \infty} \frac{\operatorname{cl}\left(a^{n}\right)}{n} .
$$

From the definition one sees that $\mathrm{cl}$ (and therefore also scl) is a characteristic function, and therefore in particular it is constant on conjugacy classes. The function scl can be extended to conjugacy classes which represent torsion elements in $H_{1}(G ; \mathbb{Z})$ by the formula $\operatorname{scl}(a)=\operatorname{scl}\left(a^{n}\right) / n$ for any positive integer $n$.

For an introduction to stable commutator length and its properties, see [4] or [8].

There is a straightforward relationship between (stable) commutator length and norm, as follows.

Lemma 4.2. Let $M$ be a 3-manifold, and $K \subset M$ a knot. Let $a \in \pi_{1}(M)$ be an element in the conjugacy class determined by the free homotopy class of $K$. Then $\operatorname{scl}(a) \leq\|K\|$.

Proof. Proposition 2.10 from [8] says that $\operatorname{scl}(a)=\inf _{S}-\chi^{-}(S) / 2 n$ where the infimum is taken over all oriented surfaces $S$ mapping to $M$ with boundary $\partial S$ mapping to $K$ with total degree $n$. If $S$ is a $p$-Seifert surface for $K$, collapsing $N(K)$ to $K$ wraps $\partial S$ around $K$ with total degree $p$. The result follows.

In general, $\operatorname{scl}(a)$ can be smaller than $\|K\|$, since the infimum in the geometric definition of $\operatorname{scl}(a)$ (in the proof of Lemma 4.2) is taken over all surfaces $S$ in $M$ which bound $K$, and not just embedded surfaces whose interior is disjoint from $K$. In other words, $\operatorname{scl}(a)$ is the best lower bound on $\|K\|$ which can be estimated from the homotopy class of $K$. If $\|K\|$ is small, then $\operatorname{scl}(a)$ is small and we will deduce information about the homotopy class of $K$ from this.

4.2. Stable commutator length in hyperbolic 3-manifolds. There are strong interactions between geometry and scl, especially in dimension 3 . The most interesting case is that of hyperbolic geometry, summarized in the following theorem:

Theorem 4.3 ([7], Theorem C). For every $\epsilon>0$ there is a constant $\delta(\epsilon)>0$ such that, if $M$ is a complete hyperbolic 3-manifold and a nontrivial $a \in \pi_{1}(M)$ has 
$\operatorname{scl}(a) \leq \delta$, then either a is parabolic, or otherwise if $\gamma$ is the unique geodesic in the free homotopy class associated to the conjugacy class of a,

$$
\operatorname{length}(\gamma) \leq \epsilon
$$

The dependence of $\delta$ on $\epsilon$ is not proper: in every finite volume hyperbolic 3manifold, conjugacy classes $a$ with $\operatorname{scl}(a)=1 / 2$ correspond to arbitrarily long geodesics. However, if $K$ is a knot with sufficiently small norm in a closed hyperbolic 3-manifold, Theorem 4.3 implies that $K$ is homotopic to a power of the core geodesic of a Margulis tube, and the length of the geodesic can be bounded from above by a constant depending on $\|K\|$.

In more detail, recall that Margulis showed that in each dimension $n$, there is a positive constant $\epsilon(n)$ so that every geodesic in a closed hyperbolic $n$-manifold of length at most $\epsilon(n)$ is simple, and is contained in an embedded solid tube whose diameter can be estimated from below by a function of length. The exact value of the biggest constant $\epsilon(n)$ with this property is not known when $n>2$, so for the sake of precision, we make the following definition.

Definition 4.4. A Margulis tube in a hyperbolic 3-manifold is an embedded solid tube of radius at least 0.531 around a simple geodesic (the core of the Margulis tube) of length at most 0.162286 .

The precise choice of constants are somewhat arbitrary, but are chosen to be compatible with the estimates obtained by Hodgson-Kerckhoff [16]; see the discussion in the next subsection.

4.3. Deformation of cone-manifold structure. We have seen in the previous subsection that if $K$ is a knot of sufficiently small norm, $K$ is homotopic into the core of a Margulis tube.

We would like to conclude in fact that $K$ is isotopic to (a cable of) the core of the tube. To do this we must use the fact that $\|K\|$ is small, not just scl of the corresponding conjugacy class in $\pi_{1}(M)$. To make use of this fact, we must study the geometry of $M-K$. In what follows we make use of some well-known results from the theory of hyperbolic cone manifolds (with non-singular cone locus). For a reference see e.g. [6] or [16].

We assume throughout this section that $M-K$ is hyperbolic. Then as Thurston already showed (see e.g. [27]), for all sufficiently small positive real numbers $\theta$, there exists a hyperbolic cone manifold $M_{\theta}$, unique up to isometry, whose underlying manifold is homeomorphic to $M$, and whose cone locus is a geodesic in the isotopy class of $K$ with cone angle equal to $\theta$.

The manifolds $M_{\theta}$ can be deformed (by increasing $\theta$ ) until one of the following happens: 
(1) The cone angle can be increased all the way to $2 \pi$, and one obtains a complete hyperbolic structure on $M$ for which $K$ is isotopic to a geodesic.

(2) The volume goes to 0 (and either converges after rescaling to a Euclidean cone manifold, or the injectivity radius goes to 0 everywhere after rescaling to have a fixed diameter).

(3) The cone locus bumps into itself (this can only happen for $\theta>\pi$ ).

In the second or third case we say that the cone manifold structure becomes singular. Under deformation, the length of the cone geodesic strictly increases. For each $\theta$, let $l(\theta)$ denote the length of the cone geodesic isotopic to $K$ in $M_{\theta}$, and let $R(\theta)$ be the radius of a maximal open embedded tube around the cone geodesic.

Following Hodgson-Kerckhoff [16] we define

$$
h(R):=3.3957 \frac{\tanh (R)}{\cosh (2 R)}
$$

which is non-negative for positive $R$, is asymptotic to 0 as $R$ goes to 0 or to $\infty$, and which has a single maximum value $\approx 1.019675$, achieved at $r \approx 0.531$.

The following is proved in $\S 5$ of [16]:

Theorem 4.5 (Hodgson-Kerckhoff). Let $h(R)$ be as above. Let $l(\theta)$ be the length of the singular geodesic in $M_{\theta}$. Then $M_{\theta}$ can be deformed (by increasing $\theta$ ) either until $\theta=2 \pi$, or until $\theta \cdot l$ is equal to the maximum of $h(R)$ (which occurs at approximately $R=0.531$ and is equal to approximately $\left.h(0.531)=h_{\max }=1.019675\right)$ and for all smaller values of $\theta$, the radius of a maximal embedded tube about the cone geodesic is at least 0.531 .

It follows that we can deform $M_{\theta}$ either all the way to $\theta=2 \pi$ with $l \leq 0.162286$ and $R \geq 0.531$, in which case $K$ is isotopic to the core of a Margulis tube, or else we can deform $M_{\theta}$ until $\theta \cdot l=h(0.531) \approx 1.019675$ for some $\theta<2 \pi$. In the second case we can estimate $l(\theta) \geq 1.019675 / 2 \pi \approx 0.162286$ and $R(\theta) \geq 0.531$. In the next few sections we will obtain a positive lower bound on the rational genus of $K$ in the second case.

4.4. 1-forms from tubes. Let $M_{\theta}$ be a cone manifold, with a cone geodesic $\gamma$ with length $l$ and tube radius $R$. Let $T$ denote an embedded tube around $\gamma$ whose radius is $R$, and let $p: T \rightarrow \gamma$ denote radial projection. Let $\phi: \gamma \rightarrow \mathbb{R} / l \cdot \mathbb{Z}$ be a parameterization of $\gamma$ so that $d \phi$ is the length form on $\gamma$. Pulling back the 1-form $d \phi$ by $p^{*}$ defines a 1 -form on all of $T$ which, by abuse of notation, we denote by $d \phi$. Let $r: T \rightarrow[0, R]$ be the function on $T$ which is equal to the radial distance to $\gamma$. Define a 1 -form $\alpha$ on $M_{\theta}$ by

$$
\alpha=d \phi \cdot(\sinh (R)-\sinh (r))
$$


on $T$, and extend it by 0 outside $T$. Let $\beta$ be a $C^{\infty}$ function on $[0, R]$ taking the value 1 in a neighborhood of 0 and the value 0 in a neighborhood of $R$, and satisfying $\left|\beta^{\prime}\right|<1 /(R-\epsilon)$ throughout $[0, R]$, for some small fixed $\epsilon$. Finally define $\alpha_{\epsilon}=\beta(r) \alpha$. Then the form $\alpha_{\epsilon}$ is $C^{\infty}$ on $M-\gamma$, and satisfies $\left\|d \alpha_{\epsilon}\right\| \leq 1+1 /(R-\epsilon)$ pointwise. Moreover, the integral of $\alpha_{\epsilon}$ along $\gamma$ is $l \cdot \sinh (R)$. For a proof of these estimates, see Lemma 4.3 from [7].

4.5. Wrapping. Suppose $K$ is a knot of sufficiently small rational genus such that $M-K$ is hyperbolic. If $M$ is hyperbolic, and $K$ is isotopic to an embedded geodesic, then $K$ is isotopic to the core of a Margulis tube (whose length may be estimated from above in terms of $\|K\|$, by Theorem 4.3). Otherwise, we can find a hyperbolic cone manifold structure $M_{\theta}$ on the underlying topological manifold $M$, with cone angle $\theta<2 \pi$ along a single cone geodesic in the isotopy class of $K$, whose length is at least 0.162286 , and is contained in an embedded tube whose radius is at least 0.531 . For each $\epsilon>0$, let $\alpha_{\epsilon}$ be a 1 -form constructed as in $\S 4.4$. Let $S$ be a good $p$ Seifert surface for $K$ in $M_{\theta}$ realizing $\|K\|$, and let $S^{\prime \prime}$ be another (possibly immersed) surface, homotopic to $S$ rel. boundary, with interior disjoint from $K$. For each $\epsilon$,

$$
p \cdot l \cdot \sinh (R)=\int_{\partial S^{\prime \prime}} \alpha_{\epsilon}=\int_{S^{\prime \prime}} d \alpha_{\epsilon} \leq \operatorname{area}\left(S^{\prime \prime}\right) \cdot(1+1 /(R-\epsilon)) .
$$

Taking $\epsilon \rightarrow 0$ we obtain an estimate

$$
l \cdot \sinh (R) \cdot R /(R+1) \leq \operatorname{area}\left(S^{\prime \prime}\right) / p .
$$

By the discussion above, we can estimate

$$
0.03131 \approx 0.162286 \cdot \sinh (0.531) \cdot 0.531 / 1.531 \leq l \cdot \sinh (R) \cdot R /(R+1) .
$$

We claim that one can find a representative surface $S^{\prime \prime}$ homotopic to $S$ rel. boundary in $M-K$, of area at most $\epsilon-2 \pi \chi(S)$ for any $\epsilon>0$. This will imply that $\|K\| \geq$ $0.03131 / 4 \pi \approx 2.491 \times 10^{-3}$.

The representative surface $S^{\prime \prime}$ is obtained by wrapping; there are two (essentially equivalent) methods to construct a "wrapped" surface: the shrinkwrapping method from [9], and the PL wrapping method from [25]. For technical ease, we use the PL wrapping method. Roughly speaking, given a surface $S$ in a hyperbolic 3-manifold and a prescribed family $\Gamma$ of geodesics, the PL wrapping technique finds a CAT $(-1)$ representative in the homotopy class of $S$, which can be approximated by surfaces homotopic to $S$ in the complement of $\Gamma$.

Our situation is analogous to, but not strictly equivalent to, the situation in [25] or [9]. In our context $\Gamma$ will be a singular cone geodesic, in the isotopy class of $K$, in a cone manifold structure on $M$; and the surface $S$ will have boundary wrapping some number of times around $\Gamma$ but interior disjoint from $\Gamma$. In fact, this extra complication 
does not add any difficulty to the argument, and we will obtain the same conclusion namely, the existence of a CAT(-1) surface which can be approximated by surfaces homotopic to $S$ (rel. boundary) in the complement of $\Gamma$. For the sake of completeness, we explain the construction in detail.

The key point of the construction is that the universal cover of $M-\Gamma$ has a metric completion which is intrinsically CAT(-1); this is Lemma 1.2 from [25]. Let $N$ denote the universal cover of $M-\Gamma$, and $\bar{N}$ its metric completion. Notice that $\bar{N}$ is obtained from $N$ by adding geodesics which project to components of $\Gamma$.

Let $T$ be a triangulation of $S$ with all vertices on $\partial S$. After an isotopy, we can assume that the map $\partial S \rightarrow \Gamma$ takes all vertices of the triangulation to distinct points in $\Gamma$.

The interior of each edge $e$ of $T$ not on $\partial S$ lifts to an open interval in $N$ whose closure is a closed interval $\tilde{e}$ in $\bar{N}$. Since $\bar{N}$ is $\operatorname{CAT}(-1)$, there is a unique geodesic $\tilde{e}^{\prime}$ in $\bar{N}$ with the same endpoints as $\tilde{e}$. This projects to a piecewise geodesic segment $e^{\prime}$ in $M$ with vertices on $\Gamma$ (note that the projection $e^{\prime}$ does not depend on the choice of the lift $\tilde{e}$ ). For each triangle $\Delta$ of $T$, we can choose lifts $\tilde{e}_{i}$ of the edges $e_{i}$ which together span a triangle $\widetilde{\Delta}$ in $\bar{N}$. After replacing each $\tilde{e}_{i}$ with a geodesic $\tilde{e}_{i}^{\prime}$, we straighten $\widetilde{\Delta}$ to a piecewise linear surface by coning one vertex to the points on the opposite edge; i.e. if $v$ is the vertex opposite $\tilde{e}_{1}^{\prime}$ (say), for each point $p$ on $\tilde{e}_{1}^{\prime}$ there is a unique geodesic in $\bar{N}$ from $v$ to $p$, and the union of these geodesics is a piecewise totally geodesic disk $\widetilde{\Delta}^{\prime}$ spanning the union of the $\tilde{e}_{i}^{\prime}$. Let $\Delta^{\prime}$ denote the projection of $\widetilde{\Delta}^{\prime}$. Then the union of the $\Delta^{\prime}$ is a $\operatorname{CAT}(-1)$ surface $S^{\prime}$ which can be approximated by surfaces whose interior is disjoint from $\Gamma$, and which are homotopic to $S$ through surfaces with interior disjoint from $\Gamma$. The approximating surfaces $S^{\prime \prime}$ can be chosen to have area as close to the area of $S^{\prime}$ as desired; since $S^{\prime}$ is CAT $(-1)$, by Gauss-Bonnet, we have area $\left(S^{\prime \prime}\right) \leq \epsilon-2 \pi \chi(S)$, as claimed.

Remark 4.6. The argument in [25] uses extra hypotheses on $S$, namely that it is incompressible and 2-incompressible rel. $\Gamma$. In fact, these hypotheses are superfluous in the case that $S$ has boundary. In fact, even when $S$ is a closed surface, one really only needs to know that $S$ contains some embedded loop which is covered by a nondegenerate infinite geodesic in $\bar{N}$.

Remark 4.7. The reader familiar with the construction of pleated surfaces in hyperbolic or CAT $(-1)$ spaces will recognize the similarity with PL wrapping.

In fact, for our applications, it is important to construct surfaces $S^{\prime \prime}$ as above when $M$ has boundary consisting of tori, and $S$ is a relative $p$-Seifert surface for $K$. There are no extra difficulties in this case. One can proceed by either of two methods. The easiest is to construct the PL wrapped surface directly. The triangulation of the relative $p$-Seifert surface can include vertices on a "cusp" boundary component; one just needs to observe that semi-infinite rays in $\bar{N}$ have geodesic representatives 
(constructed e.g. by taking limits of sequences of geodesic arcs with one endpoint going to infinity along the ray). Triangles with two vertices on a single "cusp" boundary component degenerate to a geodesic ray under straightening. The resulting surface, while non-compact, is complete, and satisfies area $\leq-2 \pi \chi$.

Alternately, one can deform the metric in a neighborhood of infinity to make it CAT(0), in such a way that each end is foliated as a metric product by Euclidean totally geodesic tori; such a metric is described explicitly in the proof of Lemma 7.12 in [9]. One obtains a compact PL wrapped surface as above with some boundary components on $\Gamma$, and some on a fixed family of Euclidean tori, one for each cusp component of $M$. The PL wrapped surface so obtained is CAT(0), and its restriction to any prescribed compact region of $M$ can be taken to be CAT(-1); in particular, we can assume that the surface is CAT $(-1)$ in the support of the 2-form $d \alpha_{\epsilon}$, so that the area of the part of the surface in the support of $d \alpha_{\epsilon}$ is at most $-2 \pi \chi(S)$, and we obtain the desired bound on $-\chi(S)$.

We have therefore obtained a proof of the following theorem:

Theorem 4.8. Let $K$ be a knot in a 3-manifold $M$, possibly with boundary consisting of a union of tori. Suppose $M-K$ is hyperbolic. Then either

(1) $\|K\| \geq 1 / 402$; or

(2) $M$ is hyperbolic and $K$ is isotopic to the core of a Margulis tube.

Moreover, if $F$ is a relative $p$-Seifert surface for $K$ in $M$, then either

(1) $\eta(F) / p \geq 1 / 402$; or

(2) $M$ is hyperbolic and $K$ is isotopic to the core of a Margulis tube.

4.6. Better estimates. In fact, though the wrapping technique explains in a direct geometric way the relationship between $\|K\|$ and the geometry of $K$ in $M$, one can obtain better estimates at the cost of appealing to some more refined technology of Agol and Cao-Meyerhoff (which we treat as a black box). For the benefit of the reader we include a sketch of a proof of the following:

Proposition 4.9. Let $K$ be a knot in a 3-manifold $M$. Suppose $M-K$ is hyperbolic. Then either

(1) $\|K\| \geq 1 / 50$; or

(2) $M$ is hyperbolic and $K$ is isotopic to the core of a Margulis tube.

Proof. Let $S$ be a $p$-Seifert surface for $K$. Let $T$ be a maximal horospherical cusp torus in $M-K$; we may regard $S$ as satisfying $\partial S \subset T$. Since $S$ is essential, Theorem 5.1 from [1] gives $|\chi(S)| \geq \ell(\partial S) / 6$ where $\ell$ denotes Euclidean length measured on $T$. Note that $\ell(\partial S)=q \ell(\sigma)$, where $q$ is the number of boundary 
components of $S$, and $\sigma$ is the boundary slope. Also, $p=q \Delta(\sigma, \mu)$, where $\Delta(\sigma, \mu)$ is the geometric intersection number of $\sigma$ with the meridian slope $\mu$. Hence

$$
|\chi(S)| / 2 p \geq \ell(\partial S) / 12 p=q \ell(\sigma) / 12 q \Delta(\sigma, \mu)=\ell(\sigma) / 12 \Delta(\sigma, \mu) .
$$

Let $A$ be the area of $T$. Then by the proof of Theorem 8.1 from [1],

$$
A \leq \ell(\sigma) \ell(\mu) / \Delta(\sigma, \mu) .
$$

Hence

$$
|\chi(S)| / 2 p \geq A / 12 \ell(\mu) \geq \sqrt{A} / 12 \ell_{N}(\mu)
$$

where $\ell_{N}(\mu):=\ell(\mu) / \sqrt{A}$ is the normalized length of $\mu$ (see [10]). By [10] one knows without hypothesis that $A \geq 3.35$. If we define

$$
C:=\sqrt{3.35} / 12(7.515) \sim 0.0203>1 / 50
$$

then we conclude

$$
|\chi(S)| / 2 p<C \text { implies that } \ell_{N}(\mu) \geq 7.515
$$

and therefore by [10] p. 410 we deduce that $M$ is hyperbolic and $K$ is isotopic to the core of a Margulis tube (i.e. a geodesic of length $\leq 0.162$ with tube radius $\geq 0.531$ ).

It is therefore probably safe to replace $1 / 402$ by $1 / 50$ throughout the sequel by appealing to Proposition 4.9 in place of Theorem 4.8 (it is, however, unlikely that $1 / 50$ is sharp).

\section{General knots}

We are now in a position to discuss the most general case of a knot $K$ in a closed, orientable 3-manifold $M$ such that $[K]$ has finite order in $H_{1}(M)$. The discussion is case-by-case, and depends on the (well-known) prime and JSJ decomposition theorems.

The first step is to consider the interaction of $(M, K)$ with the essential 2-spheres in $M$. Such spheres are treated by the following theorem.

Theorem 5.1. Let $K$ be a knot in a reducible manifold $M$. Then either

(1) $\|K\| \geq 1 / 12$; or

(2) there is a decomposition $M=M^{\prime} \# M^{\prime \prime}, K \subset M^{\prime}$ and either

(a) $M^{\prime}$ is irreducible, or 
(b) $\left(M^{\prime}, K\right)=\left(\mathbb{R} \mathbb{P}^{3}, \mathbb{R} \mathbb{P}^{1}\right) \#\left(\mathbb{R} \mathbb{P}^{3}, \mathbb{R} \mathbb{P}^{1}\right)$.

Note that in case (2) (b), $\|K\|=0$.

Proof. Let $S$ be a good $p$-Seifert surface for $K$.

First assume that $M-K$ is irreducible. Let $\Sigma$ be an essential 2-sphere in $M$, chosen so that $n=|\Sigma \cap K|$ is minimal. Since $[K]$ has finite order in $H_{1}(M)$, the algebraic intersection number of $K$ and $\Sigma$ is zero; thus $n$ is even and $>0$.

Let $P$ be the planar surface $\Sigma-$ int $N(K)$. Let $\Gamma_{S}, \Gamma_{P}$ be the intersection graphs in $\widehat{S}$ and $\Sigma$ respectively, defined by the arc components of $S \cap P$.

Case A. $(n \geq 4)$ An extended $S$-cycle is a configuration of the form depicted in Figure 3; i.e. a series of four parallel edges, whose middle pair form an ordinary $S$-cycle.

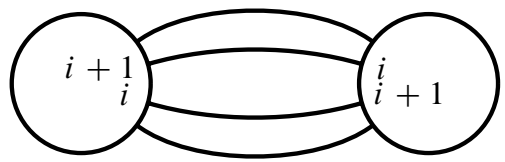

Figure 3. An extended $S$-cycle.

In this context, and with the assumption that $n \geq 4$, Lemma 2.3 from [28] says precisely that $\Gamma_{S}$ does not contain an extended $S$-cycle. Hence $\Gamma_{S}$ does not contain a family of $(n / 2+3)$ parallel edges. Let $\bar{\Gamma}_{S}$ denote the reduced graph of $\Gamma_{S}$. Since $e=p n / 2$, we can estimate $\bar{e} \geq p(n / 2) /(n / 2+2) \geq p / 2$ (because $n \geq 4)$, where $\bar{e}$ denotes the number of edges of $\bar{\Gamma}_{S}$. Hence by Lemma 3.5, we have $-\chi(S) \geq p / 6$ and therefore $\|K\| \geq 1 / 12$.

Case B. $(n=2)$ Here $P$ is an annulus, so all edges of $\Gamma_{P}$ are parallel (i.e. $\Gamma_{P}$ is a beachball).

If $\Gamma_{S}$ has a pair of parallel edges, consider an innermost such pair $e_{1}, e_{2}$, i.e. $e_{1}$ and $e_{2}$ cobound a bigon face of $\Gamma_{S}$. The corresponding edges of $\Gamma_{P}$ are necessarily parallel, and it follows that their endpoints, $x_{1}$ and $x_{2}$, say, at a given vertex of $\Gamma_{P}$ are diametrically opposite, in the sense that there are the same number of edge-endpoints in each of the two intervals around the vertex on either side of the pair $\left\{x_{1}, x_{2}\right\}$; see Figure 4 in [13]. Therefore $\Gamma_{S}$ cannot have three mutually parallel edges.

If $\Gamma_{S}$ is a beachball with exactly two edges, the argument in the proof of Proposition 1.3, case (1) from [13] shows that $\Sigma$ decomposes $(M, K)$ as a connected sum $\left(\mathbb{R} \mathbb{P}^{3}, \mathbb{R} \mathbb{P}^{1}\right) \#\left(\mathbb{R} \mathbb{P}^{3}, \mathbb{R P}^{1}\right)$, which we think of as a degenerate case of case (2) (b) in the statement of the theorem.

We may therefore assume that $\Gamma_{S}$ is not a beachball, and that no family of parallel edges in $\Gamma_{S}$ has more than two edges. Consequently the reduced graph $\bar{\Gamma}_{S}$ exists and its number of edges $\bar{e} \geq p / 2$, so Lemma 3.5 gives $\chi(S) \leq-p / 6$, and hence $\|K\| \geq 1 / 12$. This completes the analysis when $M-K$ is irreducible. 
If $M-K$ is reducible, we can write $M=M^{\prime} \# M^{\prime \prime}$ where $K \subset M^{\prime}$ and $M^{\prime}-K$ is irreducible. Let $\Sigma^{\prime}$ be a 2 -sphere in $M-K$ realizing the connected sum decomposition $M-K=\left(M^{\prime}-K\right) \# M^{\prime \prime}$. By surgering $S$ along the curves of intersection $S \cap \Sigma^{\prime}$ we get a $p$-Seifert surface $S^{\prime}$ for $K$ in $M^{\prime}$, with $-\chi^{-}\left(S^{\prime}\right) \leq-\chi^{-}(S)$. The theorem now follows from the argument in the case that $M-K$ is irreducible.

After Theorem 5.1 we may assume that $M$ is irreducible. Moreover, if $M-K$ is reducible then $K$ lies in a 3-ball in $M$ and so either $\|K\| \geq 1 / 2$ or $K$ bounds a disk (in which case $\|K\|=0$ ). We therefore assume in the sequel that both $M$ and $M-K$ are irreducible.

A closed, irreducible 3-manifold $M$ is either $S^{3}$, a lens space, an atoroidal Seifert fiber space over $S^{2}$ with three exceptional fibers, hyperbolic, or toroidal. The next theorem treats the case that $M$ is a lens space.

Theorem 5.2. Let $K$ be a knot in a lens space $M$. Then either

(1) $\|K\| \geq 1 / 24$; or

(2) K lies on a Heegaard torus in $M$; or

(3) $M$ is of the form $L(4 k, 2 k-1)$ and $K$ lies on a Klein bottle in $M$ as a nonseparating orientation-preserving curve.

Note that in case (3) we have $\|K\|=0$; this is a special case of Theorem 2.11 (3).

Proof. Let $\widehat{T}$ be a Heegaard torus in $M$. Then either (2) holds, or we can put $K$ in thin position with respect to $\widehat{T}$. Assuming the latter, let $n=|K \cap \widehat{T}|$; so $n$ is even and $\geq 2$. Let $S$ be a good $p$-Seifert surface for $K$ in $M$. We then get intersection graphs $\Gamma_{S}, \Gamma_{T}$. Thin position of $K$ and incompressibility of $S$ guarantee by Lemma 3.2 that $\Gamma_{S}$ and $\Gamma_{T}$ have no monogon disk faces.

We need a sublemma.

Sublemma 5.3. Suppose there is a pair of edges that are parallel on both $\Gamma_{S}$ and $\Gamma_{T}$. Then $M=\mathbb{R P}^{3}$, and either

(1) $\|K\| \geq 3 / 4$; or

(2) $(M, K)=\left(\mathbb{R} \mathbb{P}^{3}, \mathbb{R P}^{1}\right)$.

Proof. By Lemma 3.8 we have $(M, K)=\left(M^{\prime}, K^{\prime}\right) \#\left(\mathbb{R} \mathbb{P}^{3}, \mathbb{R}^{1}\right)$. Since $M$ is a lens space we must have $M^{\prime}=S^{3}$. If $K^{\prime}$ is trivial we have (2). Suppose $K^{\prime}$ is non-trivial. Then $\left\|K^{\prime}\right\| \geq 1 / 2$ and, since $\mathbb{R} \mathbb{P}^{1}$ in $\mathbb{R} \mathbb{P}^{3}$ is 2-trivial, Theorem 2.17 implies that $\|K\|=\left\|K^{\prime}\right\|-\frac{1}{4}+\frac{1}{2} \geq \frac{3}{4}$. 
Note that in case (2) (i.e. $(M, K)=\left(\mathbb{R} \mathbb{P}^{3}, \mathbb{R} \mathbb{P}^{1}\right)$ ) we have $\|K\|=0$.

If $\Gamma_{S}$ has $(2 n+1)$ parallel edges then by Lemma 3.7 bullet $(2)$ there are indices $i_{0}$ and $k$ so that $\Gamma_{T}$ has 5 edges labeled $\left(i_{0}, k-i_{0}\right)$. Remember that this means that there are vertices $i_{0}$ and $k-i_{0}$ in $\Gamma_{T}$ that are joined to each other by at least 5 edges. On a torus, one can find at most four embedded pairwise non-parallel arcs joining two points, that are disjoint except at their endpoints. To see this, "engulf" one of the edges by a disk, and observe that it is equivalent to show that there are at most three pairwise non-parallel essential embedded loops that intersect each other in one point; this latter fact can be shown using intersection number. Consequently we can deduce that two of the $\left(i_{0}, k-i_{0}\right)$-edges must be parallel in $\widehat{T}$.

If they are parallel in $\Gamma_{T}$ (i.e. if they cobound an embedded complementary bigon) then there is a pair of edges that are parallel in both graphs, and Sublemma 5.3 applies. If not, then the disk $D$ in $\widehat{T}$ realizing the parallelism of the two edges must contain vertices of $\Gamma_{T}$ in its interior. By Lemma 3.7 bullet (1), these vertices come in pairs $i$, $k-i$, and each pair are joined by 4 edges. An easy innermost argument in the disk $D$ shows that, for some $i$, some pair of $(i, k-i)$ edges are parallel in $\Gamma_{T}$. Hence again we get edges that are parallel in both graphs.

By Sublemma 5.3 we may therefore suppose that there is no family of $(2 n+1)$ parallel edges in $\Gamma_{S}$. If $\Gamma_{S}$ is not a beachball then the reduced graph $\bar{\Gamma}_{S}$ exists and satisfies $\bar{e} \geq e / 2 n=p n / 4 n=p / 4$. Hence $-\chi^{-}(S) \geq p / 12$ by Lemma 3.5, giving $\|K\| \geq 1 / 24$.

If $\Gamma_{S}$ is a beachball then as observed in Remark 3.4, $K$ satisfies conclusion (3) of Theorem 2.11, i.e. $K$ is the fiber of multiplicity $r$ in a Seifert fiber subspace $N$ of $M$ whose base orbifold is a Möbius band with one orbifold point, of order $r \geq 1$. Since $M$ is a lens space, $\overline{M-N}$ is a solid torus, and the meridian of this solid torus is not the Seifert fiber of $N$. Hence $M$ is a Seifert fiber space with orbifold $\mathbb{R P}^{2}$ and one orbifold point of order $k \geq 1$, where $k=r$ if $r>1$. Thus $M$ is the lens space $L(4 k, 2 k-1)$. If $r>1$ then $K$ lies on a Heegaard torus in $M$. If $r=1$ then $K$ satisfies conclusion (3).

Remark 5.4. The examples in Case B of $\$ 2.4$ show that for any $\epsilon>0$ there exists a lens space $M$ and a knot $K$ lying on a Heegaard torus in $M$ with $0<\|K\|<\epsilon$.

Remark 5.5. The bound $1 / 24$ in Theorem 5.2 is almost certainly not best possible. The smallest value of $\|K\|$ that we know of for a knot $K$ in a lens space not satisfying (2) or (3) of Theorem 5.2, comes from the following example.

Let $K^{\prime}$ be the $(-2,3,7)$-pretzel knot in $S^{3}$. Then 19 -surgery on $K^{\prime}$ gives a knot $K$ in the lens space $L(19,7)$. By Lemma $2.15,\|K\|=\left(g\left(K^{\prime}\right)-1 / 2\right) / 19=$ $(5-1 / 2) / 19=9 / 38$. Since $M-K \cong S^{3}-K^{\prime}$ is hyperbolic, $K$ does not lie on a Heegaard torus in $M$. In fact $K^{\prime}$ is a Berge knot, and so $K$ is 1-bridge in $M$.

Remark 5.6. Baker has shown in [2] that if $K$ is a knot in a lens space $M$ such that $\|K\|$ is realized by a $p$-Seifert surface $S$ with a single boundary component then 
either $K$ is 1-bridge in $M$ or $\|K\|>1 / 4$. By the proof of Lemma 2.15, the hypothesis holds for pairs $(M, K)$ that come from surgery on a knot in $S^{3}$ (or any homology sphere).

Remark 5.7. In our proof of Theorem 5.2, the argument shows that if $n>2$ then $\Gamma_{S}$ cannot have $(n+1)$ parallel edges. Hence (1) can be improved to $\|K\| \geq 1 / 12$ if (2) is weakened to say that $K$ is 0 - or 1-bridge in $M$.

The next proposition considers knots in hyperbolic 3-manifolds, possibly with boundary. The case that the complement of the knot is hyperbolic was already treated in Theorem 4.8. Here we treat the general case.

Proposition 5.8. Let $M$ be a hyperbolic 3-manifold, possibly with boundary consisting of a union of tori. Let $K$ be a knot in $M$ such that $M-K$ is irreducible, and let $F$ be a relative $p$-Seifert surface for $K$. Then either

(1) $\eta(F) / p \geq 1 / 402$; or

(2) $K$ is isotopic to a cable of a core of a Margulis tube; or

(3) $K$ is isotopic into $\partial M$.

Corollary 5.9. Let $K$ be a knot in a closed hyperbolic 3-manifold $M$. Then either

(1) $\|K\| \geq 1 / 402$; or

(2) $K$ is trivial; or

(3) $K$ is isotopic to a cable of the core of a Margulis tube.

Proof of Proposition 5.8. If $M-K$ is hyperbolic then the result follows from Theorem 4.8. So we may assume that $M-K$ is toroidal.

Since $M$ is hyperbolic, every essential torus in $M-K$ is separating, so there exists an extremal such torus, i.e. an essential torus $T$ in $M-K$ such that the component $X_{0}$ of $M$ cut along $T$ that does not contain $K$ is atoroidal. Let $N=\overline{M-X_{0}}$; thus $K$ is contained in $N$.

Let $F$ be a good relative $p$-Seifert surface for $K$ in $M$, and let $F^{\prime}=F \cap N$, $F_{0}=F \cap X_{0}$. Since $T$ is incompressible in $M-K$, and $M-K$ is irreducible, we may assume that no component of $F^{\prime}$ or $F_{0}$ is a disk. Hence $\eta(F)=\eta\left(F^{\prime}\right)+\eta\left(F_{0}\right)$.

Since $M$ is atoroidal, either $T$ compresses in $N$ or $N$ is a product $T^{2} \times I$ where $T^{2} \times\{0\}$ is a component of $\partial M$ and $T^{2} \times\{1\}=T$. In the latter case, Proposition 3.17 implies that either $\eta(F) \geq \eta\left(F^{\prime}\right) \geq p / 6$ or $K$ is isotopic into $\partial M$.

We may therefore assume that $T$ compresses in $N$. By Proposition 3.13 either $\eta\left(F^{\prime}\right) / p>1 / 12$ or $K$ is isotopic into $T$. So we may suppose the latter holds.

First suppose that $N$ is a solid torus. Let $K_{0}$ be the core of $N$. Then $K$ is a non-trivial cable of $K_{0}$. The exterior of $K_{0}$ in $M$ is $X_{0}$, which is atoroidal. Since 
$M$ is hyperbolic $X_{0}$ cannot be Seifert fibered, and therefore $X_{0}$ is hyperbolic. By Proposition $3.12\|K\| \geq 2\left\|K_{0}\right\|$. Hence either we have conclusion (1) or $\left|K_{0}\right|<$ $1 / 804$, in which case $K_{0}$ is isotopic to the core of a Margulis tube by Theorem 4.8.

If $N$ is not a solid torus then $X_{0}$ lies in a 3-ball in $M$. Since $K$ is isotopic into $T=\partial X_{0}, K$ also lies in a 3-ball, contradicting our assumption that $M-K$ is irreducible.

It remains to consider toroidal manifolds, and small Seifert fiber spaces. The small Seifert fiber spaces are treated by the following theorem. Recall that a prism manifold is a Seifert fiber space $M$ with base $S^{2}$ and three exceptional fibers of multiplicities 2, 2, $n$. Then $M$ has another Seifert fiber structure with base $\mathbb{R} \mathbb{P}^{2}$ and at most one exceptional fiber.

Theorem 5.10. Let $M$ be an atoroidal Seifert fiber space over $S^{2}$ with three exceptional fibers and let $K$ be a knot in $M$. Then either

(1) $\|K\| \geq 1 / 402$; or

(2) $K$ is trivial; or

(3) $K$ is a cable of an exceptional Seifert fiber of $M$; or

(4) $M$ is a prism manifold and $K$ is a fiber in the Seifert fiber structure of $M$ over $\mathbb{R P}^{2}$ with at most one exceptional fiber.

Note that in case (4) we have $\|K\|=0$. This is a special case of assertion (3) in Theorem 2.11.

Proof. We may assume that $X$ (i.e. $M-$ int $N(K)$ ) is irreducible.

If $X$ is hyperbolic then (1) holds by Theorem 4.8 .

If $X$ is Seifert fibered then (since $M$ is irreducible) the Seifert fibration of $X$ extends to $M$. Thus $K$ is a Seifert fiber of this fibration. If $M$ is not a prism manifold then the Seifert fiber structure on $M$ is unique and (since an ordinary fiber is a cable of an exceptional fiber) we have conclusion (3). If $M$ is a prism manifold then the two possible Seifert fiber structures on $M$ give either (3) or (4).

We may therefore assume that $X$ is toroidal. Since $M$ is irreducible and atoroidal, every torus in $X$ is separating. As in the proof of Proposition 5.8, let $T$ be an extremal essential torus in $X$, so $M=X_{0} \cup_{T} N$ where $X_{0}$ is atoroidal. Again as in the proof of Proposition 5.8, we may assume that $K$ is isotopic into $T$, and that $N$ is a solid torus, with core $K_{0}$. By Proposition 3.12, $\|K\| \geq 2\left\|K_{0}\right\|$. If $M-K_{0}$ is hyperbolic, and (1) does not hold, then $\left\|K_{0}\right\|<1 / 804$ and so $M$ is hyperbolic by Theorem 4.8, a contradiction.

Hence we may assume that $X_{0}$ is Seifert fibered. The Seifert fibration of $X_{0}$ extends to $M$, so $K_{0}$ is a fiber in the fibration of $M$, and $K$ is a non-trivial cable of $K_{0}$. Since an ordinary fiber is a non-trivial cable of an exceptional fiber, by Corollary 3.16 either $\|K\|>1 / 12$ or $K_{0}$ is an exceptional fiber. 
The toroidal case involves several subcases, which are treated in the next few propositions, culminating in Theorem 5.19.

Proposition 5.11. Let $M$ be an irreducible, toroidal 3-manifold, and let $K$ be a knot in $M$ such that $M-K$ is irreducible and atoroidal. Let $F$ be a relative $p$-Seifert surface for $K$. Then $-\chi^{-}(F) \geq p / 12$.

Proof. We may suppose that $F$ is good.

Let $\widehat{T}$ be an essential torus in $M$ such that $n=|K \cap \widehat{T}|$ is minimal (over all essential tori in $M$ ). Note that $n>0$ since $M-K$ is atoroidal. Also, the existence of $F$ shows that $p[K]=0 \in H_{1}(M, \partial M)$, which implies that $n$ is even.

Let $T$ be the punctured torus $\widehat{T} \cap(M-$ int $N(K))$ and let $\Gamma_{F}, \Gamma_{T}$ be the intersection graphs in $\widehat{F}$ and $\widehat{T}$ respectively. Note that all edges are interior edges.

We need some sublemmas.

Sublemma 5.12. If $n \geq 4$ then $\Gamma_{F}$ contains no family of more than $(n / 2+2)$ parallel edges.

Proof. Since all vertices of $\Gamma_{F}$ are of the same sign, a family of more than $(n / 2+2)$ parallel edges would contain an extended $S$-cycle. But this is impossible, again by Lemma 2.3 from [28] (compare with Case A in the proof of Theorem 5.1).

Sublemma 5.13. If $n=2$ then $\Gamma_{F}$ contains no family of 5 parallel edges.

Proof. The condition $n=2$ implies that $\Gamma_{T}$ has 2 vertices. Recall from the proof of Theorem 5.2 that on a torus one can find at most four embedded pairwise non-parallel arcs joining two points, that are disjoint except at their endpoints. Consequently if $n=2$ and $\Gamma_{F}$ contains a family of 5 parallel edges, at least two of these edges are also parallel in $\Gamma_{T}$, so Lemma 3.8 implies that $M=\mathbb{R} \mathbb{P}^{3}$, contrary to the assumption that $M$ is toroidal.

We now complete the proof of Proposition 5.11. If $F$ is not an annulus, let $\bar{e}$ be the number of edges in the reduced graph $\bar{\Gamma}_{F}$. If $n \geq 4$ then Sublemma 5.12 shows that $\bar{e} \geq p n /(n+4) \geq p / 2$. If $n=2$ Sublemma 5.13 shows that $\bar{e} \geq 2 p / 8=p / 4$. Hence $-\chi^{-}(F) \geq p / 12$ by Lemma 3.5 .

If $F$ is an annulus then by Theorem 2.11 (3) $K$ is contained as a fiber of multiplicity $r$ in a Seifert fiber submanifold $N$ of $M$, where $N$ has base orbifold a Möbius band with one orbifold point of order $r \geq 1$. In particular $N-K$ is toroidal. Since $M-K$ is atoroidal by hypothesis, $\partial N$ must be compressible in $W=\overline{M-N}$. Since $M-K$ is irreducible it follows that $W$ is a solid torus, and that the meridian of $W$ is not identified with a fiber in $\partial N$. Hence $M-$ int $N(K)$ is a Seifert fiber space over the Möbius band with at most one exceptional fiber (the core of $W$ ). Since this manifold is atoroidal by hypothesis, the core of $W$ is in fact an ordinary fiber. Then $M$ is a 
Seifert fiber space over $\mathbb{R P}^{2}$ with at most one exceptional fiber, namely $K$. But this contradicts our assumption that $M$ is toroidal.

Proposition 5.14. Let $M$ be an irreducible 3-manifold containing an essential annulus $A$. Let $K$ be a knot in $M$ and let $F$ be a good relative $p$-Seifert surface for $K$. Then either

(1) $-\chi^{-}(F) \geq p / 6$; or

(2) $K$ can be isotoped to be either disjoint from A or to lie in $\partial M$; or

(3) $F$ is an annulus.

Proof. We may assume that $F$ is good. Let $X=M-$ int $N(K)$.

Isotop $K$ so that $n=|K \cap A|$ is minimal. If $n=0$ we are done, so assume in what follows that $n>0$. Let $P=A \cap X$, and let $\Gamma_{F}, \Gamma_{P}$ be the intersection graphs in $\widehat{F}$ and $A$ respectively.

Sublemma 5.15. $\Gamma_{F}$ contains no family of $(n+1)$ parallel interior edges.

Proof. By Lemma 3.7 bullet (2), $\Gamma_{P}$ has $3\left(i_{0}, k-i_{0}\right)$-edges for some $i_{0}, k$. Two of these must be parallel in $A$ (since the union of any two is an embedded loop, and there is only one isotopy class of embedded essential loops in an annulus). If they are parallel in $\Gamma_{P}$ then we have interior edges parallel in both graphs and Lemma 3.8 readily gives a contradiction. If not, then the disk $D$ in $A$ realizing the parallelism must have vertices of $\Gamma_{P}$ in its interior. By Lemma 3.7 bullet (1) these vertices come in pairs $i, k-i$, each with 2 edges joining them. By taking an innermost such pair in $D$ we again get a pair of edges that are parallel in both graphs, a contradiction as before.

Sublemma 5.16. If $\Gamma_{F}$ contains a family of $(2 n+1)$ parallel boundary edges then $K$ is isotopic into $\partial M$.

Proof. At the vertex end of such a family every label in $\{1,2, \ldots, n\}$ appears twice, and one label appears three times. The three corresponding boundary edges in $\Gamma_{P}$ all share a common vertex; at least two must be parallel in $A$. If they are parallel in $\Gamma_{P}$, we get boundary edges parallel in both graphs, so the result follows from Lemma 3.9. If not, then the disk $D$ in $A$ realizing the parallelism must have vertices of $\Gamma_{P}$ in its interior. Each such vertex has two boundary edges coming from the given family; an innermost pair is parallel in both graphs, so we are done by Lemma 3.9.

We now complete the proof of Proposition 5.14. If $F$ is an annulus we are done. If not, then the reduced graph $\bar{\Gamma}_{F}$ exists. Let $\bar{e}_{i}, \bar{e}_{\partial}$ be the number of interior and boundary edges of $\bar{\Gamma}_{F}$. By Sublemma 5.15 each interior edge of $\bar{\Gamma}_{F}$ contributes at most $2 n$ to the sum of the valences of the vertices on $\Gamma_{F}$, and by Sublemma 5.16 we 
may assume that each boundary edge of $\bar{\Gamma}_{F}$ also contributes at most $2 n$ to this sum. Hence $\bar{e}=\bar{e}_{i}+\bar{e}_{\partial} \geq p n / 2 n=p / 2$, and the estimate in bullet (1) follows from Lemma 3.5.

In the next proposition we consider the orientable Seifert fiber spaces $D^{2}(2)$, $M^{2}(0), A^{2}(1)$ and $P^{2}(0)$, where $F^{2}(n)$ denotes a Seifert fiber space with base surface $F^{2}$ and $n$ exceptional fibers, and $D^{2}, M^{2}, A^{2}, P^{2}$ are the disk, Möbius band, annulus, and pair of pants, respectively. These, together with $S^{1} \times D^{2}$ and $T^{2} \times I$, are precisely the atoroidal Seifert fiber spaces with non-empty boundary.

Remark 5.17. This notation does not uniquely determine the space up to homeomorphism, since we do not specify the type of singular fiber.

Proposition 5.18. Let $M$ be an atoroidal Seifert fiber space of the kind $D^{2}(2)$, $M^{2}(0), A^{2}(1)$ or $P^{2}(0)$, and let $K$ be a knot in $M$. Let $F$ be a relative $p$-Seifert surface for $K$. Then either

(1) $-\chi^{-}(F) \geq p / 6$; or

(2) $K$ is isotopic into $\partial M$; or

(3) $K$ is isotopic to a cable of an exceptional Seifert fiber.

Proof. We may assume that $F$ is good and $M-K$ is irreducible.

First assume that $F$ is not an annulus. Let $A$ be an essential annulus in $M$ such that $M$ cut along $A$ is two fibered solid tori if $M=D^{2}(2)$, one fibered solid torus if $M=M^{2}(0)$ or $A^{2}(1)$, and $T^{2} \times I$ if $M=P^{2}(0)$. By Proposition 5.14 either bullet (1) or (2) holds, or $K$ can be isotoped to be disjoint from $A$.

If $M=P^{2}(0)$ and $K$ can be isotoped to be disjoint from $A$, then $K \subset T \times I$ where $T$ is a boundary component of $M$. Therefore bullet (1) or (2) holds, by Corollary 3.19.

If $M$ is one of $D^{2}(2), M^{2}(0)$ or $A^{2}(1)$ and $K$ can be isotoped to be disjoint from $A$, then $K$ is contained in a fibered solid torus $V \subset M$. Let $F^{\prime}=F \cap V$ and $F^{\prime \prime}=$ $F \cap(M-$ int $V)$. By hypothesis $M-K$ is irreducible, so $\partial V$ is incompressible in $M$-int $N(K)$. Therefore we can assume that $F^{\prime \prime}$ has no disk (or sphere) components. Hence $-\chi^{-}(F) \geq-\chi^{-}\left(F^{\prime}\right)$. Therefore by Proposition 3.13 either $-\chi^{-}(F)>p / 6$ or $K$ is a cable of $K_{0}$, the core of $V$. If $K_{0}$ is an ordinary fiber then it lies in a vertical incompressible torus $T$, so by Corollary 3.19 either bullet (1) holds or $K$ is isotopic to $K_{0}$, in which case bullet (2) holds. If $K_{0}$ is an exceptional fiber we get conclusion (3).

Finally we consider the case where $F$ is an annulus, i.e. $\Gamma_{F}$ is a beachball.

If $\Gamma_{F}$ is a beachball of the first kind then (see Theorem 2.11 (3)) $K$ is contained in a submanifold $N$ of $M$ of the form $M^{2}(0)$ or $M^{2}(1)$, as an ordinary or exceptional fiber respectively. Since $M$ is irreducible, atoroidal, and has non-empty boundary, the torus $\partial N$ is boundary parallel in $M$, and so $M \cong N$. Therefore $N$ is of the form $M^{2}(0)$, and $K$ is isotopic into $\partial M$. 
If $\Gamma_{F}$ is a beachball of the second kind, then $M=V \cup_{B} W$, where $V$ is a solid torus, $B$ is an annulus with winding number $r \geq 1$ in $V$, and $K$ is a core of $V$. If $r=1$ then $K$ is isotopic into $\partial M$. If $r>1$ then the form of $M$ implies that the (separating) annulus $B$ is essential in $M$, and hence is vertical. Therefore $K$ is an exceptional fiber in $M$.

We are finally ready to treat the case that $M$ is toroidal.

Theorem 5.19. Let $M$ be a closed, irreducible, toroidal 3-manifold, and let $K$ be a knot in $M$. Then either

(1) $\|K\| \geq 1 / 402$; or

(2) $K$ is trivial; or

(3) $K$ is contained in a hyperbolic piece $N$ of the JSJ decomposition of $M$ and is isotopic either to a cable of a core of a Margulis tube or into a component of $\partial N ;$ or

(4) $K$ is contained in a Seifert fiber piece $N$ of the JSJ decomposition of $M$ and either

(A) $K$ is isotopic to an ordinary fiber or a cable of an exceptional fiber or into $\partial N$, or

(B) $N$ contains a copy $Q$ of the twisted $S^{1}$ bundle over the Möbius band and $K$ is contained in $Q$ as a fiber in this bundle structure;

or

(5) $M$ is a $T^{2}$-bundle over $S^{1}$ with Anosov monodromy and $K$ is contained in a fiber.

Remark 5.20. If $K$ is disjoint from the hyperbolic pieces in the JSJ decomposition of $M$, the constant $1 / 402$ can be improved to $1 / 24$.

Remark 5.21. In Case (4) (B), $\|K\|=0$ (see Theorem 2.11 (3)). Also, the Seifert fibration of $Q$ induced from $N$ may be the one with base orbifold a disk with two cone points of order 2 , in which case $K$ is not a Seifert fiber in $N$.

Remark 5.22. Strictly speaking, (5) is a special case of (4) where the Seifert fiber piece $N$ is $T^{2} \times I$, but for clarity we list it separately.

Proof. We may assume that $M-K$ is irreducible. By Proposition 5.11, either $\|K\| \geq 1 / 24$ or $M-K$ is toroidal. So we may assume that $M-K$ is irreducible and toroidal.

Let $\mathcal{T}$ be a maximal disjoint union of non-parallel essential tori in $M-K$ (note that $\mathcal{T}$ is nonempty). Let $N$ be the component of $M$ cut along $\mathcal{T}$ that contains $K$. 
Then $N$ is irreducible, has boundary a non-empty disjoint union of tori, and $N-K$ is irreducible and atoroidal.

Let $S$ be a good $p$-Seifert surface for $K$ in $M$. Then $F=S \cap N$ is a relative $p$-Seifert surface for $K$ in $N$. Let $S_{0}=\overline{S-F}$. Since $\partial N$ is incompressible in $M-K$ we may assume that no component of $S_{0}$ or $F$ is a disk (or sphere). Hence $\eta(S)=\eta(F)+\eta\left(S_{0}\right)$ and so $\eta(S) \geq \eta(F)$.

If $N$ is toroidal then we are done by Proposition 5.11. If $N$ is atoroidal then $N$ is either hyperbolic or Seifert fibered. In the former case, $N$ is a piece of the JSJ decomposition of $M$, and by Proposition 5.8, either (1) or (3) holds.

Suppose $N$ is a Seifert fiber space. Since $N$ is atoroidal and has non-empty boundary it is either homeomorphic to $S^{1} \times D^{2}$ or $T^{2} \times I$, or has Seifert fiber structure $D^{2}(2), M^{2}(0), A^{2}(1)$ or $P^{2}(0)$. In the last four cases the result follows from Proposition 5.18 (to get conclusion (4) of the theorem we replace the $N$ considered here with the Seifert fiber piece of the JSJ decomposition of $M$ that contains it).

If $N=T^{2} \times I$ then by Proposition 3.17 either (1) holds or $K$ is isotopic onto $T=T^{2} \times\{1 / 2\}$. Since $T$ is an incompressible torus in $M$ there are four possibilities:

(i) $T$ is a torus in the JSJ decomposition of $M$;

(ii) $T$ is a vertical essential torus in a Seifert fiber piece of the JSJ decomposition of $M$;

(iii) $M$ is a Seifert fiber space and $T$ is horizontal;

(iv) $M$ is a $T^{2}$-bundle over $S^{1}$ and $T$ is a fiber.

If (i) holds we are done. If (ii) holds we are done by Proposition 2.24 .

Suppose (iii) holds but not (iv). Then $T$ separates $M$ into two twisted $I$-bundles over the Klein bottle. The Seifert fibering of $M$ must have Euler number 0, and $M$ is either a twisted $S^{1}$-bundle over the Klein bottle or has base orbifold $\mathbb{R} \mathbb{P}^{2}$ with two orbifold points of order 2. In both cases $M$ has an isomorphic (but non-isotopic) Seifert fibering in which $T$ is vertical.

If (iv) holds then by Remark 2.22 either $\|K\|=0$, or $\|K\| \geq 1 / 8$, or we are in Case $\mathrm{G}$ or Case $\mathrm{H}$ of $\S 2.5$. In Case G, $M$ is Seifert fibered and $K$ is a fiber. Case $\mathrm{H}$ is conclusion (5). So suppose $\|K\|=0$. Then by part (2) of Theorem 2.21 $\left(\begin{array}{ll}\alpha & \beta \\ \gamma & \delta\end{array}\right)=\left(\begin{array}{cc}-1 & n \\ 0 & -1\end{array}\right)$, with $K$ representing the first element of the corresponding basis. Thus $M$ also has the structure of an $S^{1}$-bundle over the Klein bottle with Euler number $n$, where $K$ is a fiber.

Finally, suppose $N=S^{1} \times D^{2}$. Then by Proposition 3.13, and the fact that $\mathcal{T}$ is essential in $M-K$, we may assume that $K$ is a non-trivial cable of $K_{0}$, the core of $N$. By Proposition 3.12, $\left\|K_{0}\right\|<\|K\|$.

Now repeat the whole argument with $K_{0}$ in place of $K$. We conclude that either (1) holds or

(a) $K_{0}$ is contained in a hyperbolic piece $N_{0}$ of the JSJ decomposition of $M$ and is isotopic either to a cable of a core of a Margulis tube or into $\partial N_{0}$; or 
(b) $K_{0}$ is contained in a Seifert fiber piece $N_{0}$ of the JSJ decomposition of $M$ and is isotopic either to an ordinary fiber or a cable of an exceptional fiber of $N_{0}$ or into $\partial N_{0}$, or $N_{0}$ contains a copy $Q_{0}$ of the twisted $S^{1}$ bundle over the Möbius band and $K$ is contained in $Q_{0}$ as an $S^{1}$ fiber; or

(c) $M$ is a $T^{2}$-bundle over $S^{1}$ and $K_{0}$ lies in a fiber; or

(d) $K_{0}$ is a non-trivial cable of a knot $K_{1}$.

By Proposition 3.13, $K$ cannot be a non-trivial cable of a non-trivial cable. Similarly, by Proposition 3.17, $K$ cannot be a non-trivial cable of an essential curve in an incompressible torus in $M$. This completes the proof of the theorem.

\section{References}

[1] I. Agol, Bounds on exceptional Dehn filling. Geom. Topol. 4 (2000), 431-449. Zbl 0959.57009 MR 1799796

[2] K. Baker, Small genus knots in lens spaces have small bridge number. Algebr. Geom. Topol. 6 (2006), 1519-1621. Zbl 1130.57004 MR 2253458

[3] K. Baker and J. Etnyre, Rational linking and contact geometry. In Perspectives in analysis, geometry, and topology (in honor of Oleg Viro), Progr. Math. 296, Birkhäuser/Springer, New York 2012, 19-37. Zbl 06082160 MR 2884030

[4] C. Bavard, Longueur stable des commutateurs. L'Enseign. Math. 37 (1991), 109-150. Zbl 0810.20026 MR 1115747

[5] R. Benedetti and C. Petronio, Lectures on hyperbolic geometry. Universitext, SpringerVerlag, New York 1992. Zbl 0768.51018 MR 1219310

[6] M. Boileau and J. Porti, Geometrization of 3-orbifolds of cyclic type. Astérique 272, 2001. Zbl 0971.57004 MR 1844891

[7] D. Calegari, Length and stable length. Geom. Funct. Anal. 18 (2008), no. 1, 50-76. Zbl 1155.57015 MR 2399095

[8] D. Calegari, scl. MSJ Memoirs 20, Mathematical Society of Japan, Tokyo 2009. Zbl 1187.20035 MR 2527432

[9] D. Calegari and D. Gabai, Shrinkwrapping and the taming of hyperbolic 3-manifolds. $J$. Amer. Math. Soc. 19 (2006), no. 2, 385-446. Zbl 1090.57010 MR 2188131

[10] C. Cao and R. Meyerhoff, The orientable cusped hyperbolic 3-manifolds of minimum volume. Invent. Math. 146 (2001), 451-478. Zbl 1028.57010 MR 1869847

[11] M. Culler, C. Gordon, J. Luecke and P. Shalen, Dehn surgery on knots. Ann. of Math. 125 (1987), 237-300 Zbl 0633.57006 MR 0881270

[12] D. Gabai, Foliations and the topology of 3-manifolds III. J. Differential Geom. 26 (1987), no. 3, 479-536. Zbl 0639.57008 MR 0910018

[13] C. Gordon and R. Litherland, Incompressible planar surfaces in 3-manifolds. Topology Appl. 18 (1984), 121-144. Zbl 0554.57010 MR 0769286

[14] A. Hatcher, Notes on basic 3-manifold topology. Available from the author's webpage http://www.math.cornell.edu/ hatcher/3M/3Mdownloads.html 
[15] M. Hedden, On Floer Homology and the Berge Conjecture on knots admitting lens space surgeries. Trans. Amer. Math. Soc. 363 (2011), no. 2, 949-968. Zbl 05863899 MR 2728591

[16] C. Hodgson and S. Kerckhoff, Universal bounds for hyperbolic Dehn surgery. Ann. of Math. 162 (2005), 367-421. Zbl 1087.57011 MR 2178964

[17] W. Meeks, L. Simon and S.-T. Yau, Embedded minimal surfaces, exotic spheres, and manifolds with positie Ricci curvature. Ann. of Math. (2) 116, no. 3, 621-659. Zbl 0521.53007 MR 0678484

[18] P. Ozsváth and Z. Szabó, Holomorphic disks and genus bounds. Geom. Topol. 8 (2004), 311-334. Zbl 1056.57020 MR 2023281

[19] J. Rasmussen, Lens space surgeries and $L$-space homology spheres. Preprint, arXiv:0710.2531 [math.GT].

[20] Y. Rieck, Heegaard surfaces and Dehn fillings: $g(M)+1 \leq t(X) \leq g(M)$. PhD thesis, UT Austin 1997.

[21] Y. Rieck, Heegaard structures of manifolds in the Dehn filling space. Topology 39 (2000), 619-641. Zbl 0944.57013 MR 1746912

[22] M. Scharlemann, Smooth spheres in $\mathbb{R}^{4}$ with four critical points are standard. Invent. Math. 79 (1985), 125-141. Zbl 0559.57019 MR 0774532

[23] R. Schoen and S.-T. Yau, Existence of incompressible minimal surfaces and the topology of three-dimensional manifolds with non-negative scalar curvature. Ann. of Math. (2) 110, no. 1, 127-142. Zbl 0431.53051 MR 0541332

[24] P. Scott, The geometries of 3-manifolds. Bull. London Math. Soc. 15 (1983), no. 5, 401-487. Zbl 0561.57001 MR 0705527

[25] T. Soma, Existence of ruled wrappings in hyperbolic 3-manifolds. Geom. Topol. 10 (2006), 1173-1184. Zbl 1130.57025 MR 2255495

[26] W. Thurston, A norm for the homology of 3-manifolds. Mem. Amer. Math. Soc. 59 (1986), no. 339, i-vi and 99-130. Zbl 0585.57006 MR 0823443

[27] W. Thurston, Geometry and topology of 3-manifolds. Available at http://library.msri.org/books/gt $3 \mathrm{~m} /$.

[28] Y.-Q. Wu, The reducibility of surgered 3-manifolds. Topology Appl. 43 (1992), no. 3, 213-218. Zbl 0817.57019 MR 1158868

Received December 10, 2009

Danny Calegari, Department of Mathematics, Caltech, Pasadena, CA 91125, U.S.A.

E-mail: dannyc@its.caltech.edu

Cameron Gordon, Department of Mathematics, University of Texas, Austin TX, 78712, U.S.A.

E-mail: gordon@math.utexas.edu 